\title{
The Effect of Impingement on Transitional Behavior in Underexpanded Jets
}

\author{
Jennifer A. Inman, ${ }^{*}$ Paul M. Danehy, ${ }^{\dagger}$ Robert J. Nowak, ${ }^{*}$ and David W. Alderfer ${ }^{\S}$ \\ NASA Langley Research Center, Hampton, VA, 23681-2199
}

\begin{abstract}
An investigation into the development of flow unsteadiness in impinging axisymmetric underexpanded jets has been conducted at NASA Langley Research Center. The study has examined the effect of an impingement target placed at various distances and angles on transitional behavior of such jets. Two nozzles, with exit Mach numbers of 1.0 and 2.6, were used in this investigation. Planar laser-induced fluorescence of nitric oxide (NO PLIF) has been used to identify flow unsteadiness and to image transitional and turbulent flow features. Measurements of the location of the onset of various degrees of unsteady flow behavior have been made using these PLIF images. Both qualitative and quantitative comparisons are presented to demonstrate the observed effects of impingement and flow parameters on the process of the transition to turbulence. The presence of the impingement target was found to significantly shorten the distance to transition to turbulence by up to a factor of approximately three, with closer targets resulting in slightly shorter distance to transition and turbulence. The location at which the flow first exhibits unsteadiness was found to have a strong dependence on the presence and location of key flow structures. This paper presents quantitative results on transition criteria for free and impinging jets.
\end{abstract}

\section{Nomenclature}

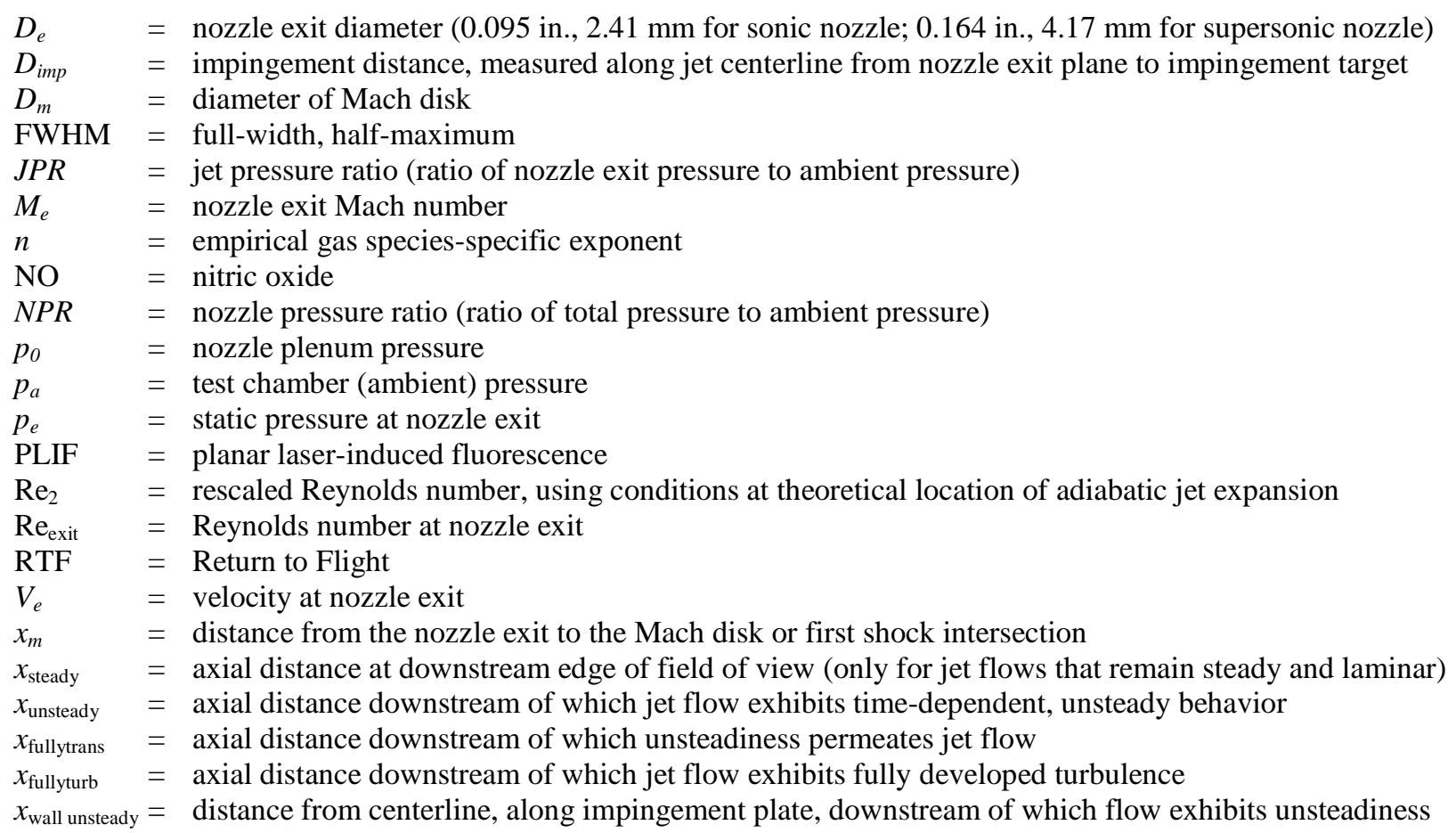

\footnotetext{
${ }^{*}$ Research Scientist, Advanced Sensing and Optical Measurement Branch, MS 493, AIAA Member.

${ }^{\dagger}$ Research Scientist, Advanced Sensing and Optical Measurement Branch, MS 493, AIAA Associate Fellow.

* Research Scientist, Aerothermodynamics Branch, MS 408A, AIAA Member.

${ }^{\S}$ Research Scientist, Advanced Sensing and Optical Measurement Branch, MS 493.
} 


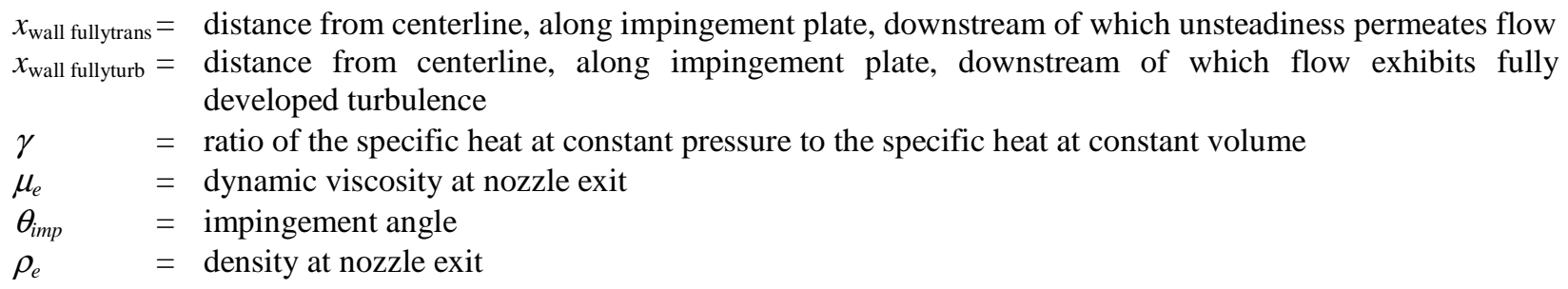

\section{Introduction}

$\mathrm{T}$ HE tests described in this paper were designed to create a simplified simulation of the flow through a hole in the surface of a hypersonic aerospace vehicle and the subsequent impingement of the flow on internal vehicle structures, with the objective of quantifying free and impinging jet transition parameters. These tests were conducted in support of the Space Shuttle Orbiter Aerothermodynamics Working Group as part of NASA's Shuttle Return to Flight (RTF) effort. Planar laser-induced fluorescence (PLIF) of nitric oxide (NO) was used to visualize the flow and to quantify the effect of impingement on the process of transition to turbulence in underexpanded jets. PLIF images show the size and location of flow structures. Additionally, the laminar or turbulent state of these flows can be ascertained from these images. The difficult task of accurately predicting the transition from laminar to turbulent flow remains an unsolved problem for many ranges of conditions. Furthermore, CFD (computational fluid dynamics) turbulence models are generally based upon the properties of boundary layer flow along surfaces rather than free shear flow, and are often based on data taken in the incompressible (with a Mach number less than about 0.3 ) regime, ${ }^{1}$ whereas the present experiments are in the compressible flow regime. The measurements of the unsteady, transitional, and/or turbulent jet flow field locations resulting from these tests can therefore potentially be used to determine where turbulence models should be implemented in CFD simulations in addition to providing data on the size, location, and other spatial characteristics of flow structures for comparison with CFD results.

The pioneering work into the experimental study of underexpanded jets was reported by Love et al. ${ }^{2,3}$ and involved schlieren flow visualization of jets exhausting into atmospheric pressure. Impinging jet flows were studied extensively by Donaldson et al., ${ }^{4,5}$ including pitot pressure surveys and surface pressure measurements. But the motivation behind these and many subsequent studies is almost universally cited as the study of rocket plumes impinging on the ground, or exhaust from the engine of a V/STOL (vertical/short takeoff and landing) vehicle. As a result of the pressures involved in such flows, related studies were almost all conducted at high Reynolds numbers (over 30,000) where the flows were fully turbulent. Much of the available literature did not apply directly to the kind of problem and flow regimes which were of interest in the present investigation-laminar and transitional flow through a breach in the heatshield of the Orbiter along a hypersonic-to-subsonic and rarefied-to-atmospheric reentry flight trajectory. Table 1 presents a comparison of several of the relevant operating conditions covered by previous studies. The subscript $e$ indicates conditions at the nozzle exit, while $a$ indicates ambient conditions and imp indicates quantities associated with impingement. See Ref. 6 for a more exhaustive list of impinging jet studies.

The absolute pressures, Reynolds numbers, and pressure ratios that we would expect for a breach in the Orbiter differ from the impinging jet flows in the literature in the following ways. The absolute pressures in the current investigation are lower, the Reynolds numbers are generally lower, the pressure ratios are generally higher, and the impingement distances (in terms of the number of nozzle diameters) are larger. This is because the flows of interest in the literature generally were at atmospheric pressures, were modeled after the flow through engines that were designed to have relatively low exit pressure ratios, and had rocket-to-ground impingement distances of a few nozzle diameters. Although a rocket taking off has a virtually infinite range of impingement distances, the investigations in the literature were only concerned with the near-field impingement flow within a few nozzle diameters, where the surface heating, ground erosion, and effects on overall thrust or lift would be greatest. Additionally, the angle of impingement is an important variable in the present experiment but only normal impingement is generally relevant in rocket launch studies. 


\begin{tabular}{|c|c|c|c|c|c|c|c|}
\hline & $\mathrm{M}_{\mathrm{e}}$ & $\mathrm{Re}_{\text {exit }}$ & $\begin{array}{l}J P R \\
\left(p_{e} / p_{a}\right)\end{array}$ & $\begin{array}{l}\mathrm{p}_{\mathrm{a}} \\
(\mathrm{atm})\end{array}$ & $\begin{array}{l}D_{\text {imp }} \\
/ D_{\mathrm{e}}\end{array}$ & $\begin{array}{l}\theta_{\text {imp }} \\
(\text { deg })\end{array}$ & $\begin{array}{l}\text { Type(s) of } \\
\text { measurements }\end{array}$ \\
\hline $\begin{array}{l}\text { Alvi \& lyer } \\
\text { (1999) }\end{array}$ & 1 & $1.9 \mathrm{E} 6$ & 2.6 & 1 & $1.6-2$ & 90 & $\begin{array}{l}\text { PIV, shadowgraph, } \\
\text { acoustic, surface } \\
\text { pressure }\end{array}$ \\
\hline $\begin{array}{l}\text { Donaldson \& } \\
\text { Snedeker } \\
(1971)\end{array}$ & $0.57,1$ & $\begin{array}{l}1.9 \mathrm{E} 5- \\
1.3 \mathrm{E} 6 \\
\end{array}$ & $1-3.57$ & 1 & $\begin{array}{l}1.96- \\
39.1\end{array}$ & $15-90$ & $\begin{array}{l}\text { Pitot } \& \text { surface } \\
\text { pressure, grease } \\
\text { streak, heat transfer }\end{array}$ \\
\hline $\begin{array}{l}\text { Kim et al. } \\
(2003)\end{array}$ & 1 & $\begin{array}{l}\text { 9.7E5- } \\
\text { 2.7E6 }\end{array}$ & $1.1-3.7$ & 1 & $1.6-2$ & 90 & $\begin{array}{l}\text { Computational (3D } \\
\text { unsteady NS) }\end{array}$ \\
\hline $\begin{array}{l}\text { Lamont \& } \\
\text { Hunt (1980) }\end{array}$ & 2.2 & $\begin{array}{l}4.0 \mathrm{E} 6- \\
6.7 \mathrm{E} 6 \\
\end{array}$ & $1.2,2$ & 1 & $0.75-15$ & $30-90$ & $\begin{array}{l}\text { Shadowgraph, } \\
\text { surface pressure }\end{array}$ \\
\hline $\begin{array}{l}\text { Love \& Lee } \\
\text { (1958) }\end{array}$ & $1-3$ & $\begin{array}{l}1.7 \mathrm{E} 5- \\
5.7 \mathrm{E} 7\end{array}$ & $\begin{array}{l}0.25-19 \\
60- \\
41,820\end{array}$ & $1,5 e-5$ & NA & NA & $\begin{array}{l}\text { Schlieren, method of } \\
\text { characteristics } \\
\text { calculations }\end{array}$ \\
\hline Stitt (1961) & $1-9.85$ & $\begin{array}{l}3.7 \mathrm{E} 4- \\
2.7 \mathrm{E} 7\end{array}$ & \begin{tabular}{|l|}
$545-$ \\
$1.5 \mathrm{E} 5$
\end{tabular} & $4.7 \mathrm{E}-4$ & $0.4-40$ & 90 & $\begin{array}{l}\text { Schlieren, surface } \\
\text { pressure, surface } \\
\text { erosion }\end{array}$ \\
\hline $\begin{array}{l}\text { Present work } \\
\text { and Refs. 9, } \\
10, \& 14-16\end{array}$ & $1,2.6$ & $\begin{array}{l}170- \\
3.6 \mathrm{E} 4\end{array}$ & $1-38$ & \begin{tabular}{|l|}
$1.3 \mathrm{E}-3$ \\
-0.094
\end{tabular} & $\begin{array}{l}10.5- \\
39.5\end{array}$ & $\begin{array}{l}90,60 \\
45\end{array}$ & $\begin{array}{l}\text { PLIF, surface } \\
\text { pressure }\end{array}$ \\
\hline
\end{tabular}

Table 1. Comparison of previous underexpanded jet studies with the present investigation. The quantities compared here are, from left to right: nozzle exit Mach number, Reynolds number based on nozzle exit conditions, jet (nozzle-exit-to-ambient) pressure ratio, ambient pressure into which jet exhausts, impingement distance in nozzle diameters, impingement angle, and type of study and/or measurements.

The previous studies - both experimental and computational—of transition and turbulence in these flows were performed almost exclusively with the aim of understanding the acoustic phenomena (i.e., jet noise) associated with such flows. In these type of studies, measurements of turbulence are generally made using hotwire anemometry, and the data are in the form of inferred velocity oscillations as a function of time. These data are then Fourier transformed to extract the frequency spectrum of the pressure oscillations. Truly turbulent flows are characterized by broadband spectra, or nearly broadband: all modes of oscillation are present in the flow, from the spatial scale of the flow itself down to the molecular dissipation scale (also called the "Kolmogorov scale"). Such data were often obtained at points in a single plane, such as the plane of an impingement target, or at points along a single line, such as the centerline of the flow. Because of this, the state of the jet upstream of or between measurement locations was either unknown or had to be inferred. This is a limitation of any single-point measurement approach. Flow visualization data have been sparse, and are almost exclusively path-averaged. Thus, limited direct observation of spatial jet structures had been made prior to the current studies. Understanding the stability characteristics of such flows was a primary motivation behind this work.

Past studies have used flow visualization to examine underexpanded jet structure in the near field (that is, the flow field in close proximity to the nozzle exit). The vast majority of these studies used schlieren, a path-averaged technique that is sensitive to strong density gradients in the flow. A series of studies by Lamont and Hunt ${ }^{7}$ examined the relationship between flow structures and characteristics of pressure profiles on an impingement target at various angles. They acquired extensive sets of schlieren and shadowgraph images showing great detail of the complicated shock structures associated with both free and impinging jets. They were able to show that the minimum and maximum surface pressures corresponded to specific impinging flow structures. Schlieren and shadowgraph, however, would not be adequate in the present investigation as they are poorly suited for low pressure/low density environments such as those expected in the current experiments, and cannot spatially resolve transitional and turbulent flow structures. Conversely, since this study was completed, electron beam fluorescence has been shown to be another potential method for investigating low Reynolds number jet flows. ${ }^{8}$ The technique works well at low densities but not at high densities.

We have developed an image processing technique-involving calculation of the standard deviation of the intensity in PLIF images in order to aid in the identification of unsteadiness, transition, and turbulence-with the aim of empirically determining criteria governing the onset of turbulence. Jet scaling parameters were used to 
define a rescaled Reynolds number that incorporates the influence of a varying pressure ratio. Measurements of the axial location associated with various stages in the transitional process will be presented for sonic and supersonic impinging jet cases.

This paper is the latest in a series of reports on this sequence of testing. Earlier papers reported on the transition to turbulence in free (non-impinging) underexpanded sonic jets ${ }^{9}$; compared three free, steady laminar, sonic nozzle cases with computational fluid dynamics (CFD) results ${ }^{15}$; explored the relation between flow structures and impingement surface pressure measurements for steady laminar, impinging, supersonic nozzle cases ${ }^{16}$; and identified instability modes of transition and the importance of flow structures in contributing to flow unsteadiness for free, supersonic nozzle cases. ${ }^{10}$ This paper will concentrate on unsteady, impinging jets for both sonic and supersonic nozzle cases.

\section{Experimental Methods}

\section{A. Facility and Hardware}

Tests were conducted at NASA Langley Research Center using the test section of the 15-Inch Mach 6 Air Tunnel as a vacuum chamber. Nitrogen or helium seeded with $0.5 \%$ nitric oxide was plumbed into a heated stainless steel plenum, through a nozzle, and into the vacuum chamber. Two different nozzles were used: the first-a converging nozzle with the exit at the smallest diameter, or throat, and hereafter referred to as the sonic nozzle-had a nominal exit Mach number of 1; the second-a converging/diverging nozzle, hereafter called the supersonic nozzle — had a nominal exit Mach number of 2.6. Mass flow controllers controlled the flow rates, which indirectly controlled the plenum pressure upstream of the nozzle.

A $10.16 \mathrm{~cm}$ (4.00 in.) diameter impingement disk was positioned at various distances and angles downstream of the nozzle exit. The center of this disk included 32 pressure taps. Pressure data from a laminar subset of impinging jet cases can be found in Ref. 16. From the camera's viewing angle, the jet flow was from left to right.

\section{B. Model Configuration Parameters}

For the majority of cases, the impingement disk was oriented normal to the jet axis (which is defined to be a $90^{\circ}$ impingement angle). Two other configurations included oblique impingement angles of $45^{\circ}$ and $60^{\circ}$. For these cases, the target was tilted clockwise, as viewed from the camera viewing angle, about the horizontal axis perpendicular to the jet axis so that the laser sheet illuminated the entire impingement surface. The hardware was designed in such a way that impingement distance was continuously variable from 0 to 6 in. $(15.24 \mathrm{~cm})$; the distances chosen for these tests ranged from 1.0 in. $(2.54 \mathrm{~cm})$ to $5.0 \mathrm{in} .(12.7 \mathrm{~cm})$. Minimum impingement angle at a given impingement distance was limited geometrically by the radius $(5.08 \mathrm{~cm}, 2.00 \mathrm{in}$.) of the impingement disk. In practice, changing impingement distance or angle required approximately a half day of down time, whereas changing the exit Reynolds number or jet pressure ratio (parameters which are defined below) generally required less than five minutes. As a consequence, the full test matrix includes many different Reynolds numbers and jet pressure ratios, but relatively few impingement distances and impingement angles (see the test matrix in Table 2).

For each hardware configuration, two flow parameters were varied: the exit Reynolds number $\left(\operatorname{Re}_{e x i t}\right)$ and the jet pressure ratio $(J P R)$. $\mathrm{Re}_{e x i t}$ was defined in terms of the density $\rho_{e}$, velocity $V_{e}$, diameter, $D_{e}$, and dynamic viscosity $\mu_{e}$ at the nozzle exit, as given by (1).

$$
\operatorname{Re}_{e x i t}=\frac{\rho_{e} V_{e} D_{e}}{\mu_{e}}
$$

$\mathrm{Re}_{\text {exit }}$ was varied by changing the mass flow rates and nozzle plenum temperature. In practice, electrically heated tape was wrapped around the stainless steel plenum and then covered with insulation. This method of heating the gas provided only coarse control of the gas plenum temperature, and day-to-day or run-to-run variations in plenum temperature contributed to scatter in the Reynolds number component of the data. The $J P R$ was defined as the ratio of the static pressure at the nozzle exit, $p_{e}$, to the ambient pressure in the test section, $p_{a}$, according to (2), and was varied by changing the test section pressure for a given Reynolds number (and therefore, a fixed $p_{e}$ ).

$$
J P R=\frac{p_{e}}{p_{a}}
$$

Note that the nozzle exit pressure was calculated based on measured plenum pressure, $p_{0}$, and nominal nozzle exit Mach number, $M_{e}$, using isentropic flow equations. This relationship is stated explicitly in (3), where $\gamma$ is the ratio of the specific heats ${ }^{1}$ : 


$$
p_{e}=p_{0}\left(1+\frac{\gamma-1}{2} M_{e}^{2}\right)^{\gamma /(1-\gamma)}
$$

Much of the literature uses nozzle pressure ratio $(N P R)$ as a defining jet characteristic —instead of $J P R$-where $N P R$ is defined as the ratio of the plenum (i.e. total) pressure to the ambient pressure $\left(N P R=p_{0} / p_{a}\right)$. To convert $J P R$ to $N P R$, simply multiply the $J P R$ by $p_{0} / p_{e}$. For a sonic nozzle $\left(M_{e}=1\right)$, this factor is 1.89 ; for the supersonic nozzle used in this study $\left(M_{e}=2.6\right)$, this factor is 19.95 .

As reported in Ref. 9 for sonic nozzle, free jet results, when measured distances to transition were graphed in terms of Reynolds number alone, no obvious correlation was observed. This led to the conclusion that jet behavior was a function not only of Reynolds number, but of jet pressure ratio as well. This same conclusion was extended to supersonic nozzle, free jet results in Ref. 10.

Scaling laws described by Yüceil and Ötügen ${ }^{11}$ provide a means of redefining the Reynolds number to include the effects of $J P R$. Conditions at the nozzle exit serve as initial conditions for defining an equivalent jet. Yüceil and Ötügen assume that the jet defined by these initial conditions is allowed to expand adiabatically until the pressure in the jet reaches the ambient pressure. At this point (or more precisely, because this is an axisymmetric flow, at this plane) - which they call location 2-all of the flow conditions are recalculated. The expressions for rescaled density $\rho_{2}$, velocity $V_{2}$, temperature $T_{2}$, and jet diameter $D_{2}$ can be found in Refs. 11 and 14 . These yield an expression for the rescaled Reynolds number in terms of $J P R$ and nozzle exit conditions. This expression is given in Eq. 4:

$$
\begin{gathered}
\operatorname{Re}_{2}=\frac{\rho_{2} V_{2} D_{2}}{\mu_{2}}=\operatorname{Re}_{e x i t} \frac{\rho_{2}}{\rho_{e}} \frac{V_{2}}{V_{e}} \frac{D_{2}}{D_{e}} \frac{\mu_{e}}{\mu_{2}} \\
=\operatorname{Re}_{e x i t} \frac{\left(\gamma M_{e}^{2} J P R+J P R-1\right)^{1 / 2}\left(2^{1 / 2} \gamma^{2} M_{e}^{2} J P R^{2}\right)^{n}}{\left.2 \gamma M_{e}^{2} J P R(\gamma+J P R-1)-(\gamma-1)(J P R-1)^{2}\right]^{n+1 / 2}}
\end{gathered}
$$

where $n$ is an empirical constant, taken from Ref. 12, which aims to account for the effect of temperature on dynamic viscosity. The value of $n$ is specific to the gas species in question: 0.67 for $\mathrm{N}_{2}, 0.78$ for NO, and 0.666 for He. The calculations presented herein have used measured mass flow rates of the supply gases to compute a weighted average for $n$ based on the gas composition in the plenum for each run.

\section{PLIF Flow Visualization Technique}

The PLIF laser system included a tunable Nd:YAG-pumped dye laser followed by doubling and mixing crystals. The resulting output, at $226.256 \mathrm{~nm}$, was tuned to excite the strongly fluorescing spectral lines of NO near the $\mathrm{Q}_{1}$ branch band head. ( $Q$ denotes a change in rotational quantum number equal to zero. The subscript 1 indicates that, in both the upper and lower states involved in the transition, the rotational quantum number, $J$, that includes electronic spin is related to $N$ by $J=N+1 / 2$, where $N$ is the quantum number for total angular momentum, excluding electronic and nuclear spin. See Refs. 13 and 14 for a more thorough explanation of the molecular spectroscopic notation used to describe the nitric oxide transitions probed in these experiments.) Optics formed the beam into a laser sheet that was $100 \mathrm{~mm}$ wide $\mathrm{x} \sim 0.2 \mathrm{~mm}$ thick (FWHM) in the measurement region. Fluorescence was imaged onto a gated, intensified CCD (charge-coupled device) at a viewing angle normal to the laser sheet. Images were acquired at $10 \mathrm{~Hz}$ with a $1 \mu$ s camera gate and a spatial resolution of between 3 and 7 pixels $/ \mathrm{mm}$, depending on the required field of view for a given hardware configuration. This system is described in detail in Refs. 9, 14, and 15.

\section{Identification of Unsteady Flow Behavior Using PLIF Images \\ 1. Image Processing}

An overview of the image processing procedures that have been used for the PLIF images presented herein is given in the following sections. These procedures are described more fully in Refs. 9, 14, and 16. After a background image is subtracted from each smoothed PLIF image, significant left-to-right variations in intensity remain. These variations are due to the left-to-right variations in both the laser sheet intensity and laser wavelength. To correct for these effects, the images are divided by a laser sheet profile. As final steps in the image processing procedure, images were sometimes cropped, a mask was applied to cover the nozzle hardware in order to eliminate any persistent glints or scattered light, and a false-color mapping was applied. Absolute intensity in the images has been arbitrarily adjusted to span the dynamic range of the false color table that has been applied. This false color mapping has been applied to enhance contrast and to increase sensitivity in regions of low signal. 
The spatial resolution was determined by imaging either a ruler (in earlier runs) or a dotcard (in later runs) positioned in the same plane as the laser sheet. (A dotcard is simply a two-dimensional rectangular array of square dots of known size and with known spacing. We have generally used black dots printed on white card stock, affixed to a rigid plate of, for example, corrugated aluminum.) The optical access in these experiments permitted perpendicular viewing of the measurement plane and no significant perspective or lens distortion was found in the images. For experimental configurations where optical access is more limited and perpendicular viewing is not possible, dotcard imaging makes it possible to correct for perspective and lens distortion. ${ }^{17}$

\section{Standard deviation images}

After background subtraction and laser sheet correction, an average image was created from all the images from a given run (each run consisting of either 100 or 200 single-shot images). A standard deviation image was then created in the following manner. Each single shot image was subtracted from the averaged image. The difference in intensity was divided by the average intensity, resulting in a percentage (rather than an absolute) difference in intensity. This percentage difference in intensity at each pixel was squared, and the squares were summed over all images in the run. Finally, the sum of the squares was divided by the number of images and the square root was taken. The resulting image provides a map of the flow, highlighting regions of large percentage variations in intensity. In general, steady laminar flows will have relatively consistent shot-to-shot intensity at a given location in the flow, resulting in percentage standard deviations of less than about 30\%. Unsteady flows, by contrast, will have regions where the intensity varies in each image, resulting in typical percentage standard deviations of between about $35 \%$ and $100 \%$. A drawback of this technique is that it tends to highlight variations in regions where there are steep gradients in image intensity in the average image (e.g. along jet boundaries) more prominently than variations in regions of comparable intensity (e.g. in the jet core).

\section{Experimental Results}

Table 2 shows the range of conditions and hardware configurations for which data were taken during the impinging jet study. The table lists the number of cases that were studied for each combination of hardware configuration and type of PLIF imaging that was investigated in these tests. For each flow visualization case, at least 100 single-shot images were acquired. Note that because Reynolds numbers and jet pressure ratios were calculated based on nozzle exit conditions, and because the exit pressure for a supersonic nozzle are lower than those of a sonic nozzle for the same total pressure, the range of $R_{\text {exit }}$ and $J P R$ for supersonic nozzle cases is less than for sonic nozzle cases. The controlling limitation on the achievable range of test conditions was the inability of the facility to achieve steady chamber pressures below 1 or 2 Torr $(0.02-0.04 \mathrm{psi} ; 130-270 \mathrm{~Pa})$.

\section{A. Characteristic Flow Structures}

PLIF images have shown that free jet cases having the same $J P R$ exhibit similar flow features (e.g. size and location of shock structures), so long as the cases under consideration are all laminar. ${ }^{9}$ For sonic nozzle cases, flows can be divided into two major groups: those with a repeating diamond shock structure (seen for flows with $J P R$ less than about 3), and those with a barrel shock structure, a Mach disk (a disk-shaped normal shock wave), and a streamwise high-velocity jet boundary (seen for flows with $J P R$ s greater than about 3). ${ }^{9}$

Figure 1 contains a PLIF image of a highlyunderexpanded jet flow issuing from a sonic nozzle, with major flow features identified. For cases with the Mach 2.6 supersonic nozzle, the division between repeating shock patterns and barrel shock/Mach disk patterns happens at a $J P R$ of about 3.5 or 4 . For smaller $J P R \mathrm{~s}$, a repeating pattern analogous to the diamond shock pattern is evident, with a bead-like pattern of alternating spatial minima (high-pressure nodes) and maxima (low-pressure antinodes). Above a $J P R$ of about 4 , high pressure nodes are no longer evident, and as $J P R$ continues to increase, the spatial oscillations in

\begin{tabular}{|r|c|c|c|c|c|c|c|c|}
\hline & \multicolumn{6}{|c|}{ Supersonic Noz. } & \multicolumn{5}{|c|}{ Sonic Nozzle } \\
\hline Impingement configuration & $F V / V I$ & $V$ & $P$ & $\rho$ & $F V / V I$ & $V$ & $P$ & $\rho$ \\
\hline $2.54 \mathrm{~cm}\left(1.0^{\prime \prime}\right) @ 90^{\circ}$ & & & & & 25 & & 5 & \\
\hline $4.45 \mathrm{~cm}\left(1.75^{\prime \prime}\right) @ 90^{\circ}$ & 24 & 56 & 23 & & 25 & & 1 & 1 \\
\hline $6.35 \mathrm{~cm}\left(2.5^{\prime \prime}\right) @ 90^{\circ}$ & 22 & 36 & 23 & & 24 & & & \\
\hline $6.35 \mathrm{~cm}\left(2.5^{\prime \prime}\right) @ 60^{\circ}$ & 19 & & & & & & & \\
\hline $9.53 \mathrm{~cm}\left(3.75^{\prime \prime}\right) @ 90^{\circ}$ & 24 & & & & 24 & & & \\
\hline $9.40 \mathrm{~cm}\left(3.7^{\prime \prime}\right) @ 45^{\circ}$ & 24 & & & & & & & \\
\hline $12.7 \mathrm{~cm}\left(5.0^{\prime \prime}\right) @ 90^{\circ}$ & 24 & & & & & & & \\
\hline$\infty$ (free jet) & 110 & 33 & & 12 & 182 & 34 & 17 & \\
\hline $\mathrm{Re}_{\text {exit }}$ & $300-23,000$ & $200-22,000$ \\
\hline Re & $300-18,000$ & $200-18,000$ \\
\hline JPR & \multicolumn{3}{|c|}{$1-32$} & \multicolumn{2}{|c|}{$1.7-38$} \\
\hline NPR & \multicolumn{2}{|c|}{$11-634$} & \multicolumn{3}{|c|}{$3-71$} \\
\hline
\end{tabular}

Table 2. Test matrix for impinging jet cases. FV/VI = flow visualization and volume imaging; $\mathrm{V}=$ velocity-sensitive imaging; $P=$ pressure-sensitive imaging; $\rho=$ density-sensitive imaging. 
the high-velocity jet boundary gradually decrease. These jets with larger $J P R$ s exhibit a modified barrel shock structure-elongated into a more egg-like shape than its comparable sonic jet counterpartwith a Mach disk and a streamwise high-velocity jet boundary.

Figure 2 schematically illustrates the difference between flows with high and low JPR, as well as between sonic and supersonic jet flows. Three dimensions have been labeled in these schematic diagrams: the nozzle diameter, $D_{e}$; the diameter of the Mach disk, $D_{m}$, if present; and the distance, $x_{m}$, between the nozzle exit and the

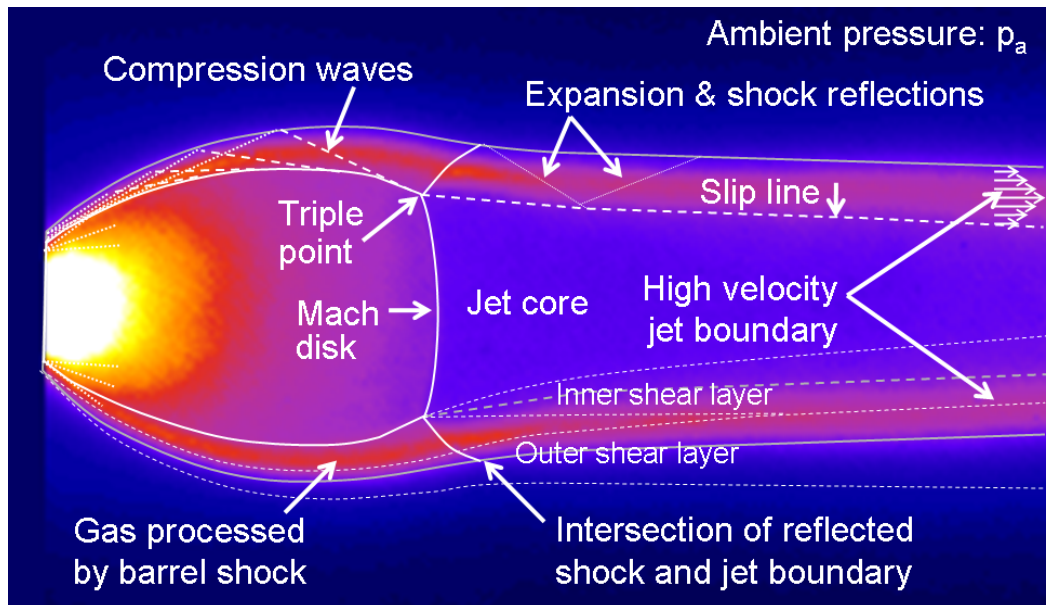

Figure 1. Highly-underexpanded sonic jet flow structures. PLIF image is from Run 56 with $J P R=29.1$ and $\operatorname{Re}_{2}=1,782$.

Mach disk and triple point or between the nozzle exit and the shock intersection at the end of the first shock cell. Note that for sonic nozzle flows, the length of the first shock cell is approximately the same as the jet diameter. Then note that for supersonic nozzle flows, this distance is longerapproximately two jet diameters.

Additional flow features, including some of those associated with the impingement region, are illustrated in Fig. 3. Both images are of supersonic nozzle flows. The figure on the left is of a high $J P R$ flow with a Mach disk and shows the impingement of the jet boundary on the impingement target. Note that the flow upstream of the Mach disk is supersonic, but immediately downstream of this normal shock, the flow is

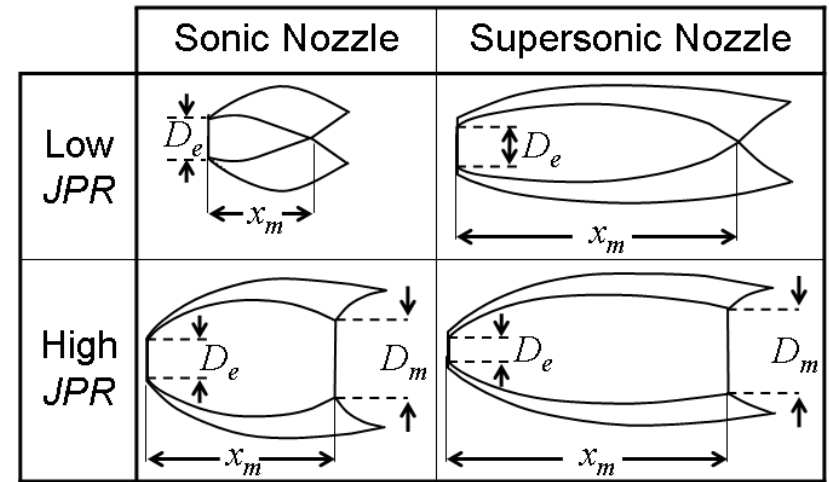

Figure 2. Typical structure of first shock cell for four categories of underexpanded jet flows. subsonic. By contrast, the flow that passes through the oblique barrel shock and the oblique reflected shock emanating from the triple point may remain supersonic. The existence of subsonic regions of the flow has significant implications for the propagation of pressure variations and instabilities in the flow; such fluctuations cannot travel upstream in supersonic regions of the flow, but will do so in subsonic regions. The image on the right is of a lower jet pressure ratio flow and exhibits repeating shock cells but no Mach disk. We call the widest part of

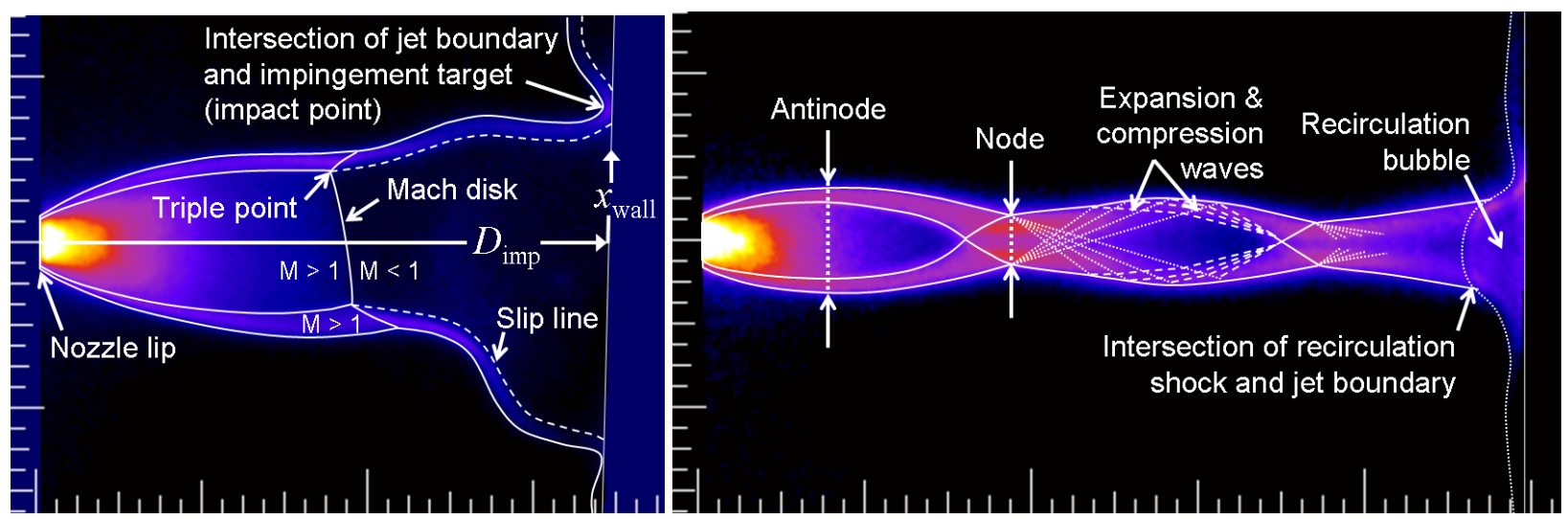

Figure 3. Impinging jet flow structures. For reference, these single-shot PLIF images are from Runs 366 (left) and 541 (right) with JPR of 9.2 and 3.6, respectively. The smallest hash marks on the scales are $1 / 16^{\text {th }}$ of an inch. 
each shock cell an antinode (or flow maximum) and the narrowest part of the flow between each shock cell a node (or flow minimum). In this particular case, a recirculation bubble has formed between the jet and the impingement target, near the third antinode. The intersection of the jet boundary with the recirculation shock is labeled. For many flows, we found the presence or absence of a recirculation bubble to be a stable and repeatable flow characteristic. However, as reported previously, ${ }^{14,16}$ for a more limited set of conditions, such a bubble was found to be unstable, with the flow exhibiting staging behavior. That is, infinitesimal perturbations or fluctuations would cause the flow to discontinuously and abruptly jump between one stage (e.g. with a recirculation bubble) and another (e.g. without a recirculation bubble). See Refs. 14 and 16 for a more thorough discussion of staging behavior in impinging jets.

\section{B. Effect of Impingement Distance and Angle on Transition}

The presence of the impingement target was found to promote the development of instabilities. In some cases, this meant that unsteadiness was observed to begin prior to the impingement region, and further upstream than in the comparable free jet case. In other cases, the impingement region was found to amplify existing instabilities or to generate new instabilities. This effect is illustrated for one $\mathrm{Re}_{\text {exit }}$ and $J P R$ combination in Figure 4. Note that very little instability (as evidenced in the standard deviation images) is observed in the free jet case, but that the presence of the impingement target results in the amplification of instabilities. Also note that instabilities do not appear to originate from random locations within the flow, but rather from locations corresponding to shock intersections and/or the nozzle exit. As reported in Refs. 10 and 14, these are regions of high vorticity and seem to act to amplify

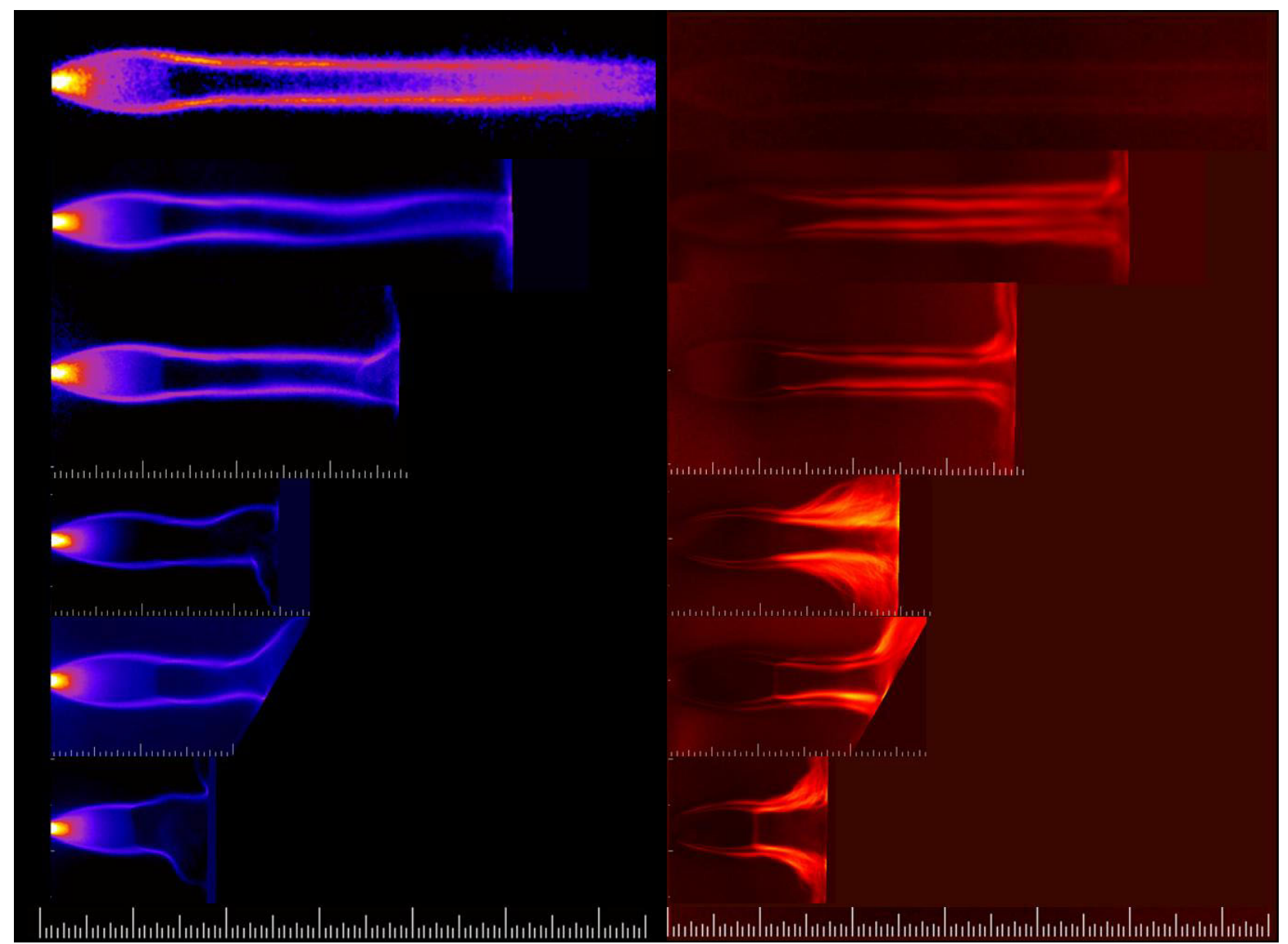

Figure 4. Unsteadiness as a function of impingement configuration for high JPR. The top images are of a free (non-impinging) jet. The smallest hash marks on the scales are $1 / 16^{\text {th }}$ of an inch. Jet pressure ratios were all about 11, with the exception of the bottom images, for which it was 9.2. The rescaled Reynolds numbers for these six runs were between 2,500 and 3,000. For reference, from top to bottom, these images are from Runs 229, 602, 577, 533, 552 , and 366 . 
underlying modes of flow instability. They are also locations adjacent to presumably subsonic regions (which could serve as pathways for the propagation of acoustic waves) and supersonic regions of the flow, the combination of which may result in an accumulation or focusing of flow perturbations at these locations.

Figure 5 shows a similar set of images for a similar exit Reynolds number, but a smaller jet pressure ratio. In these cases, the situation is somewhat different. Notice that instabilities are observed in the free-jet case. The presence of the impingement target seems to alter, not so much the location of the onset of transitional behavior, but rather the specific flow structures that result, and perhaps the intensity of the resulting fluctuations in the flow. A possible exception is in the bottom set of images, where the impingement distance is less than the distance downstream of the nozzle exit at which instabilities are first seen in the free-jet case. In the free-jet case, instabilities are seen to originate at an antinode of the flow. In the case with the shortest impingement distance, the intersection of the jet boundary with the impingement target appears to be the origin of the observed instabilities.

For the $45^{\circ}$ and $60^{\circ}$ impingement configurations, the majority of the flow was found to be deflected through the oblique angle (up the plate in the arrangements as shown, where the top of the plate is further away from the nozzle exit plane than the bottom of the plate), with little flow being deflected backward through the steeper acute angle (down the plate). In general, the normal $\left(90^{\circ}\right)$ configurations were found to be more perturbative than the oblique configurations. Figure 6 illustrates this. Seven normal impingement cases and corresponding $60^{\circ}$ cases at similar jet pressure ratios are presented in Fig. 6 . The intensity in the standard deviation images can be seen to be greater for the normal impingement cases, indicating a stronger influence on jet flow transition. Note especially the differences in the instability modes that are evident in the oblique cases as compared to the normal impingement

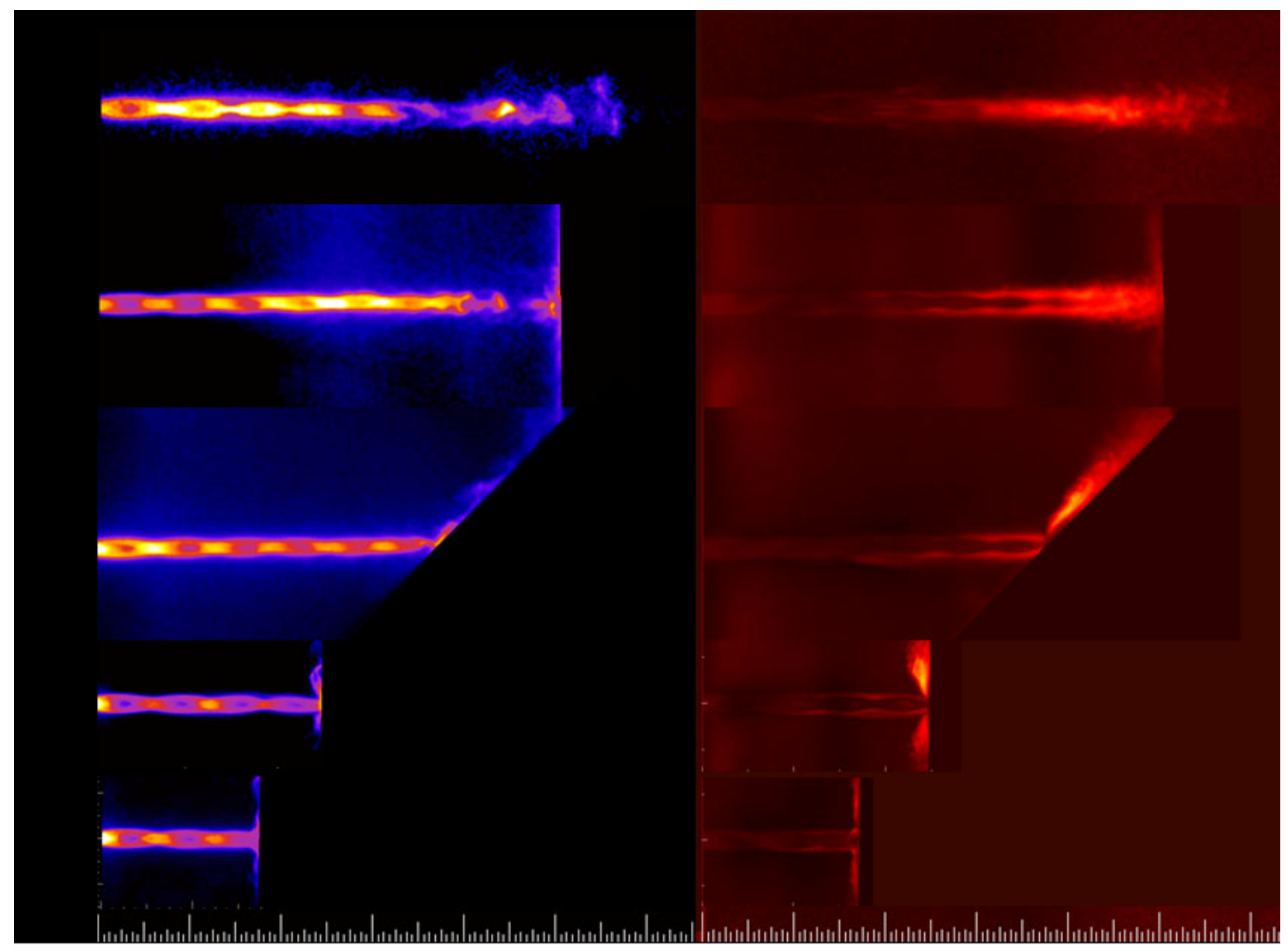

Figure 5. Unsteadiness as a function of impingement configuration for low $\boldsymbol{J P R}$. The top images are of a free (non-impinging) jet. The smallest hash marks on the scales are $1 / 16^{\text {th }}$ of an inch. Jet pressure ratios were all about 1.7, with the exception of the top images, for which it was 2.0. The rescaled Reynolds number for these five runs was between 5,800 and 6,400. For reference, from top to bottom, these images are from Runs 248, 619, 643, 549, and 380 . 

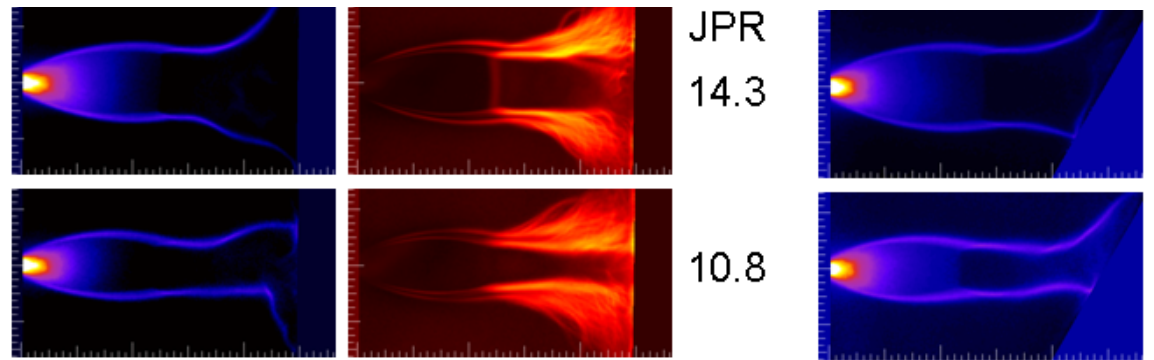

10.8
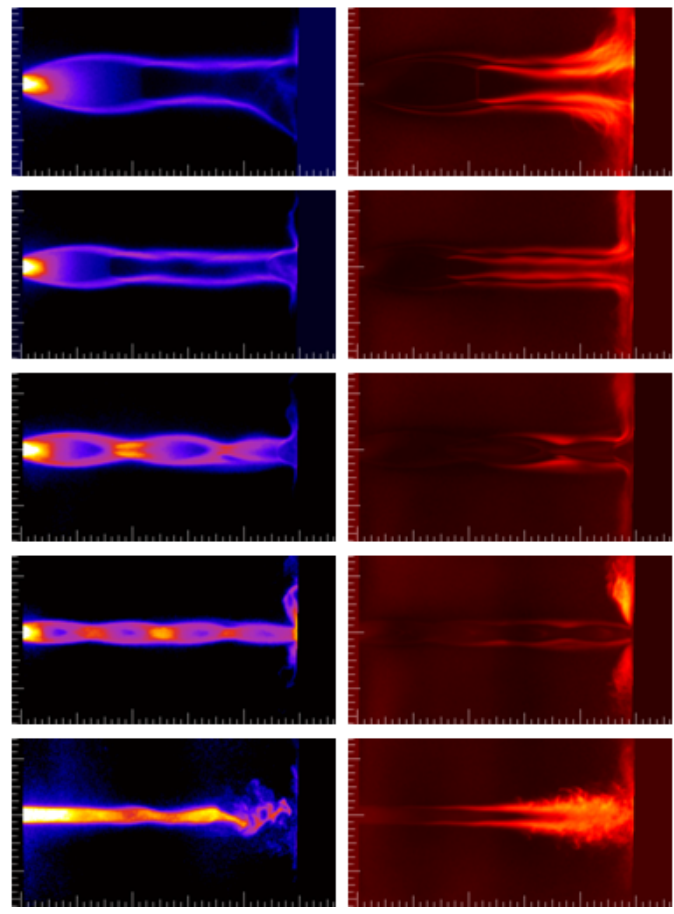
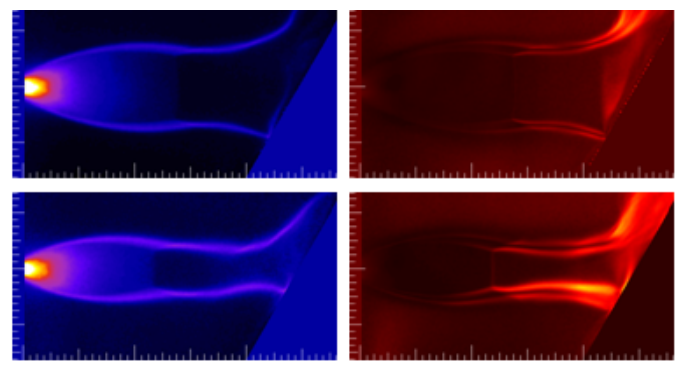

JPR

18.5

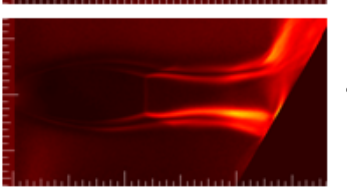

11.1
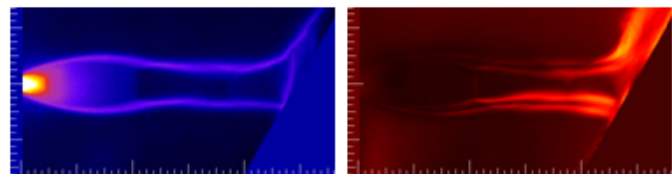

8.5
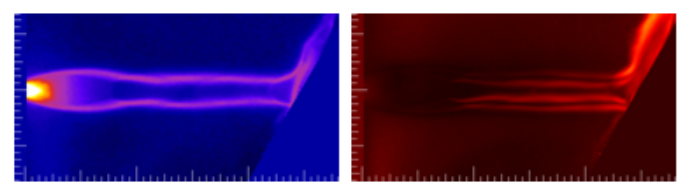

5.0
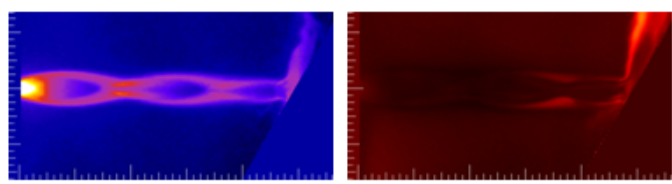

3.4
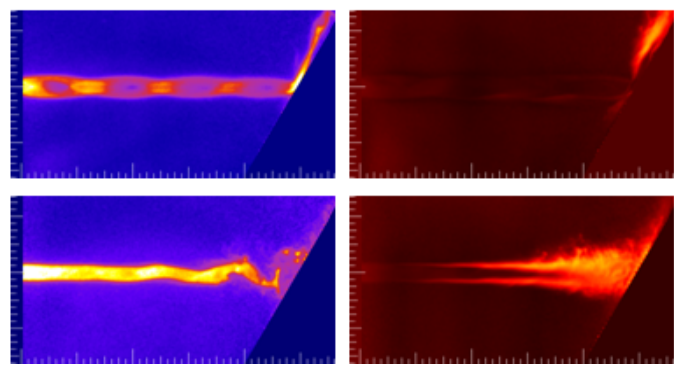

0.8

Figure 6: Effect of impingement angle for supersonic flows over a range of pressure ratios. The jet pressure ratios are indicated to the right of the standard deviation images. The exit Reynolds numbers are all approximately 7,500, with the exception of the topmost set of images at the highest JPR. For these runs, $\operatorname{Re}_{\text {exit }}$ is 12,505 for the $90^{\circ}$ case and 14,975 for the $60^{\circ}$ case. The rescaled Reynolds numbers, however, range from 2,600 to $8,300\left(\operatorname{Re}_{2}\right.$ for the highest JPR runs is about 4,000).

cases, as well as the division between flows with and without Mach disks that occurs at a JPR of around 3.5 or 4 . At the higher jet pressure ratios, the jet boundary appears to flap around its mean position. By comparing the standard deviation images, it seems that the spatial extent of this flapping is greater in the normal cases than in the oblique cases, indicating a more perturbative effect in the normal cases. At intermediate jet pressure ratios (1.7 and 3.4), the growth of instabilities stemming from flow maxima can be seen for both normal and oblique cases. Finally, at the lowest jet pressure ratio $(0.8)$, both normal and oblique cases exhibit sinuous mode behavior.

\section{Effect of Reynolds Number on Transition}

For free (non-impinging) jet supersonic nozzle cases, we have found that flows with rescaled Reynolds numbers of less than approximately 2,600 remained steady within the measurement region. For impinging supersonic nozzle flows at the impingement distances and angles of this study, this rescaled Reynolds number was found to decrease to approximately 1,800. In general, increases in Reynolds number were seen to be associated with an increase in the level of unsteadiness of a given jet flow, as expected. However, one characteristic that was not anticipated by the authors was the tendency of flow features (e.g. the shock intersections at the triple point) to be the primary determining factor in the location of the onset of unsteadiness, with increasing Reynolds number reducing the distance from the onset of unsteadiness to the onset of transition and turbulence. This effect is explored quantitatively in the next section, but can be seen qualitatively in Figs. 7 and 8. In Fig. 7, the overall degree of 

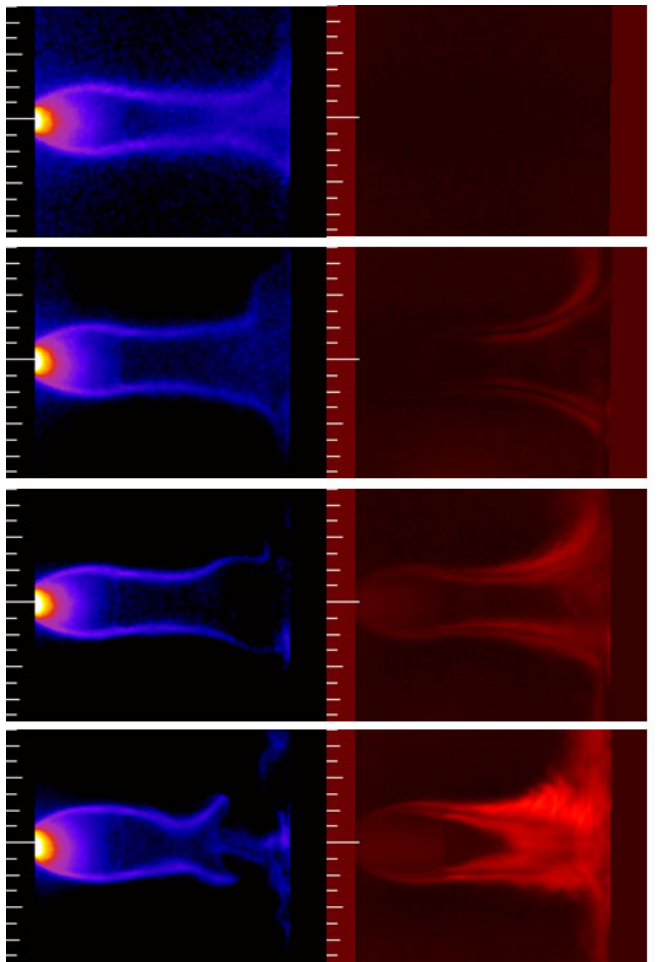

Figure 7. Effect of Reynolds number (for a $J P R$ of approximately 15). Sonic nozzle. $\mathrm{D}_{\text {imp }}=2.5 \mathrm{~cm}$ (1.0 in.); $\theta_{\text {imp }}=90^{\circ}$. $\operatorname{Re}_{2}=$ 1,$289 ; 2,069 ; 3,642$; and 6,748 and run numbers are $748,752,759$, and 765 . (top to bottom).
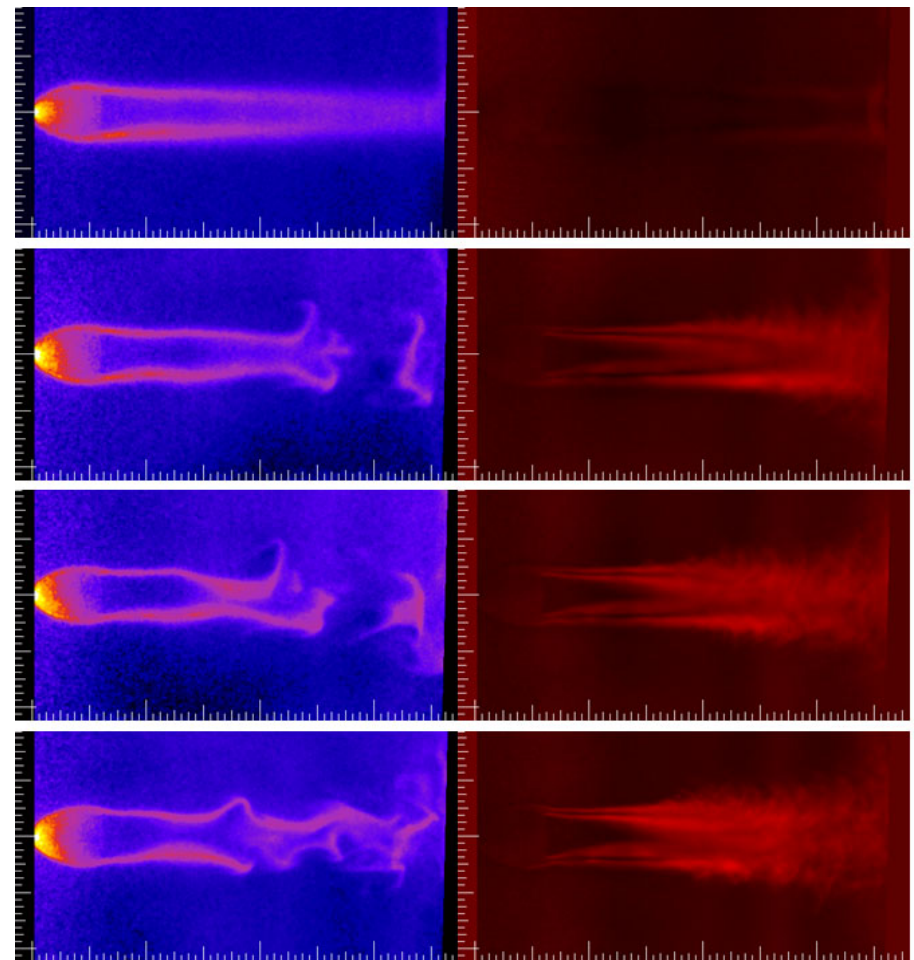

Figure 8. Effect of Reynolds number (for a $J P R$ of approximately 20). Rescaled Reynolds numbers, $\mathrm{Re}_{2}$, for these four sonic nozzle runs with $\mathrm{D}_{\text {imp }}=6.4 \mathrm{~cm}$ (2.5 in.) and $\theta_{\text {imp }}=90^{\circ}$ are (top to bottom) 1,$793 ; 3,027 ; 3,763$ and 4,903. For reference, these are run numbers 700, 704, 708, and 710 .

unsteadiness is seen to increase from the top pair of images (which is from a run with an $\mathrm{Re}_{2}$ below the proposed $\mathrm{Re}_{2}$ steady threshold of 1,800 ) to the bottom pair of images. In Fig. 8, only the smallest level of unsteadiness can be seen in the top image pair (which is from a run with an $\operatorname{Re}_{2}$ very near the proposed $\operatorname{Re}_{2}$ steady threshold of 1,800 ), just upstream of the impingement plate. In the second pair of images, unsteady behavior is seen to originate near the Mach disk, at the triple point and the reflected shock/jet boundary intersection. In the third and fourth image pairs, unsteadiness continues to originate from these same locations, but the location of the onset of transitional behavior is seen to move upstream and the degree of unsteadiness is seen to escalate with increasing Reynolds number.

\section{Quantitative Treatment of Effect of Impingement}

Conceptually, a given flow can be divided into four types of flow-steady laminar (no time-dependent flow features), unsteady laminar (some time-dependent behavior), transitional (with the spatial scale of flow features decreasing), and turbulent (where all spatial scales are present in the flow)—not all of which will necessarily be present under a given set of conditions. The standard deviation images described above have been used to distinguish between each of these flow types. For simplicity, we have defined boundaries between these four regions (based on these four flow types) as a function solely of the axial coordinate, $x$. This means, for example, that in the region we have called the unsteady laminar region, even though the core of the jet is unsteady laminar, the edges of the jet actually may be beginning to exhibit transitional behavior. The criteria that have been used to define the distances to these boundaries between these regions are defined below. Figure 9 gives and example of two cases_one steady laminar and one fully turbulent—with these distances indicated on both a single-shot PLIF flow visualization image and the corresponding standard deviation image for each run. 

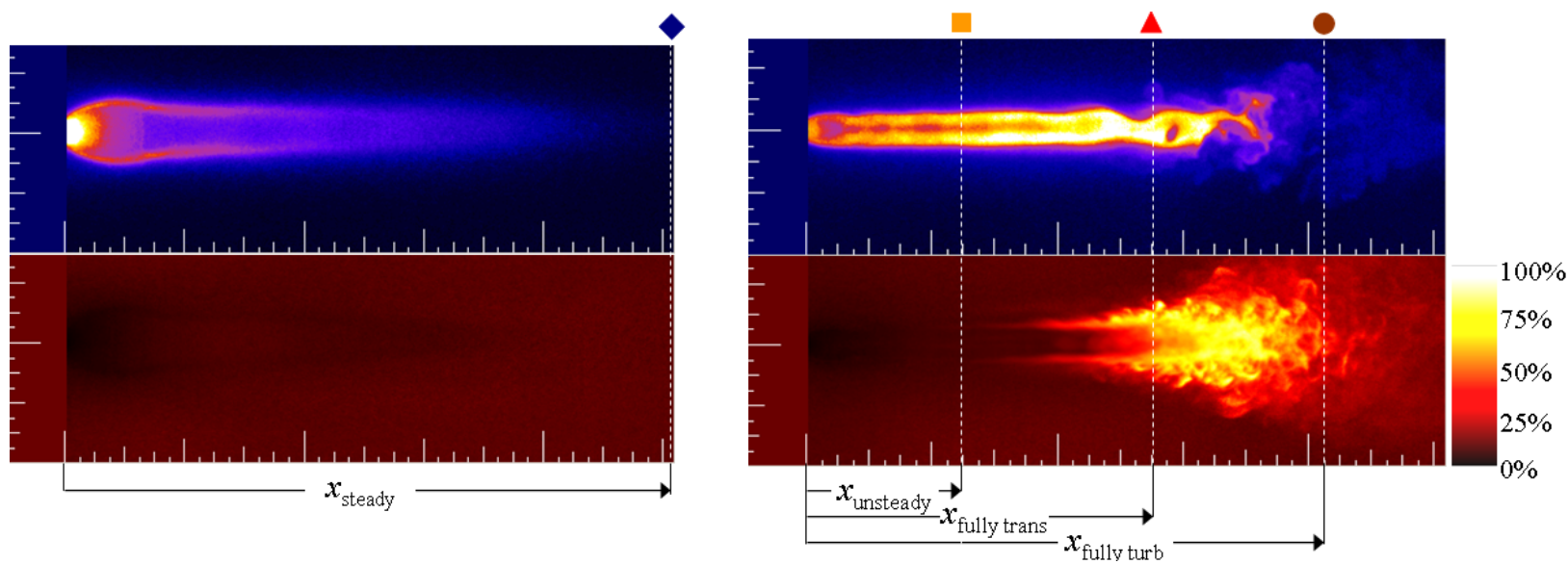

Figure 9. Quantitative transition lengths defined. Color scale indicates the standard deviation of the intensity at a given pixel as a percentage of the average intensity at that pixel. Note that the symbols (diamond, square, triangle, circle) at the top of the figure correspond to those shown on all graphs. For reference, these images are from sonic nozzle free jet Runs 49 (left) and 32 (right), with $J P R=15.8$ and 3.9, and $\operatorname{Re}_{2}=1,419$ and 3,953, respectively.

$x_{\text {steady }}$-Only flows which remain entirely steady laminar in every PLIF image corresponding to a given run will have this label applied to them. In such cases, the furthest downstream location is marked as $x_{\text {steady }}$; in other words, this distance is equal to the maximum distance between the nozzle exit and the downstream edge of the field of view of the camera for a given run. In standard deviation images, this means that the intensity remains low and relatively constant across the image. We have chosen to define this parameter so that some meaningful information about transition can be extracted from cases that did not exhibit any degree of unsteady behavior. In such cases, we cannot determine the distance to the boundary between steady and unsteady regions, but rather can only stipulate that such a flow either remains steady indefinitely, or that if such a boundary exists, it must lie downstream of our field of view. Because of this, $x_{\text {steady }}$ does not represent a distance to a boundary between two regions, unlike the remaining parameters defined below, but rather a lower limit on the distance over which a given flow remains steady.

For cases in which the flow exhibits some degree of unsteadiness, there will not be a location marked as $x_{\text {steady }}$. In such cases, we are able to specify the location of the boundary between steady and unsteady behavior.

$x_{\text {unsteady }}$ - This is defined to be the distance to the boundary between the steady laminar and unsteady lminar regions of the flow. In other words, it is the downstream location at which the flow first exhibits unsteady behavior. All locations upstream of this point are effectively time independent; all locations downstream exhibit some level of unsteadiness. In standard deviation images, this location is characterized by an increase in intensity above the background (noise) level.

$x_{\text {fully trans }}$ - This is the distance to the boundary between the laminar unsteady and transitional regions of the flow. In other words, it is defined to be the location downstream of which all of the jet, including the jet core, is exhibiting transitional behavior. Note that - although some transitional behavior may be observed in the region we are calling laminar unsteady, especially along the boundaries of the jet - in the present work we have elected to wait until the entire flow is transitional to label a flow transitional. In standard deviation images, it is the location where the intensity in the core of the flow has risen above the background level.

$x_{\text {fully turb }}$-This is the distance to the boundary between the transitional and turbulent regions of the flow. In other words, it is the location downstream of which the flow can be said to be fully turbulent.

In addition to $x_{\text {steady }}, x_{\text {unsteady }}, x_{\text {fully trans, }}$, and $x_{\text {fully turb }}$, distances along the impingement target were recorded. Because the flow becomes a wall jet after impingement, these distances were called $x_{\text {wall unsteady }}, x_{\text {wall }}$ fully trans, and

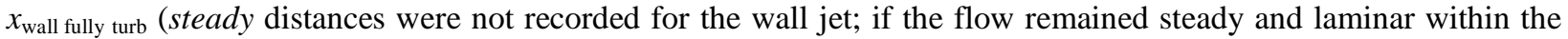
field of view in an impinging case, the impingement distance was recorded for $x_{\text {steady }}$ ). These distances along the impingement target were measured from the intersection of the jet centerline with the target. Before plotting the measured points, the distance along the plate was added to the impingement distance, to indicate the total distance the jet had traveled to get to the location where, for instance, it became fully transitional. Locations upstream of the impingement target are indicated by solid symbols, whereas those for locations along the impingement target surface are indicated by open symbols. 


\section{Effect of Impingement Angle}

Figures 10 and 11 are plots of these measured distances to unsteady flow behavior for oblique $\left(45^{\circ}\right.$ and $\left.60^{\circ}\right)$ impingement angles. They show the importance of flow features in determining the location of the onset of flow unsteadiness. Due to time and budget constraints, only one impingement distance was investigated for each of these angles (however, note that the impingement distance was different for each impingement angle). When the distances at which the flow became unsteady, transitional, or turbulent are graphed in terms of rescaled Reynolds number versus equivalent-jet diameters, several trends emerge. Consider the solid yellow squares in Fig. 10, which
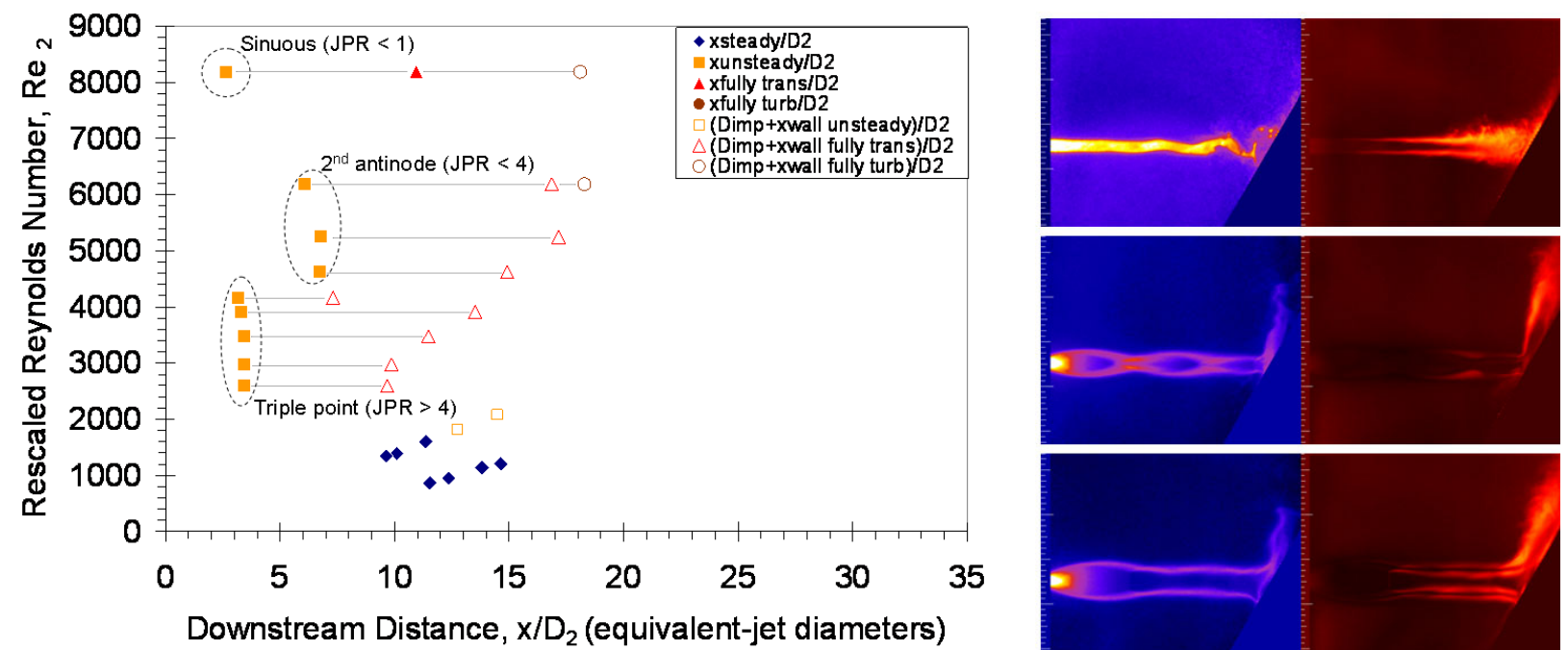

Figure 10. Oblique $\left(6^{\circ}\right)$ impingement angle (supersonic nozzle): Distances to unsteady jet behavior. $D_{\text {imp }}=$ $6.4 \mathrm{~cm}$ (2.5 in.). Distances are given in units of equivalent-jet diameters. Open symbols indicate locations along the face of the impingement plate. Light grey lines connect data points associated with the same run. The PLIF flow visualization and standard deviation images on the right show, from top to bottom, examples of sinuous, $2^{\text {nd }}$ antinode, and triple point instabilities. For reference, these are runs 570, 567, and 562 with rescaled Reynolds numbers of 8,188; 4,623; and 3,473, respectively. Exit Reynolds numbers are 7,597; 7,588 and 7,571.
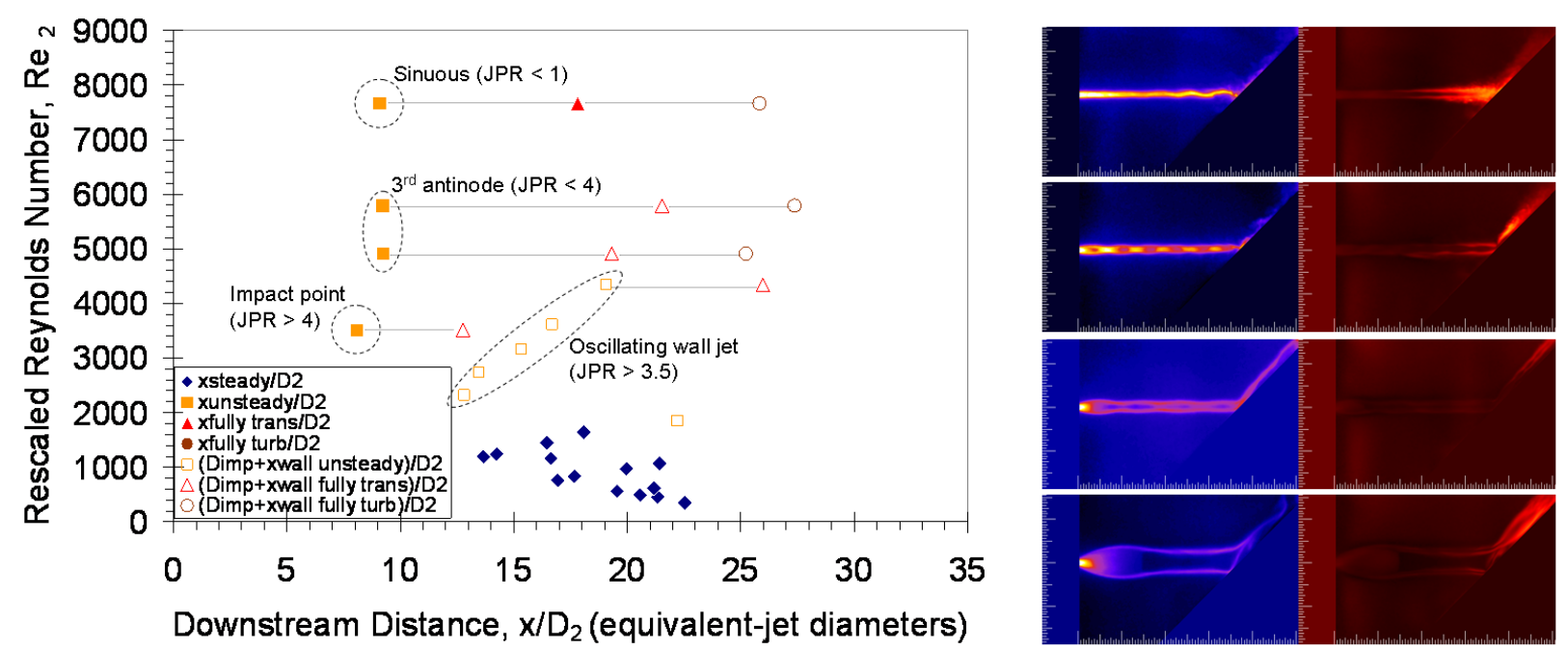

Figure 11. Oblique $\left(45^{\circ}\right)$ impingement angle (supersonic nozzle): Distances to unsteady jet behavior. Data are for an impingement distance of $9.5 \mathrm{~cm}$ (3.75 in.). Distances are in units of equivalent-jet diameters. Open symbols indicate locations along the face of the impingement plate. Light grey lines connect data points associated with the same run. The PLIF flow visualization and standard deviation images on the right show, from top to bottom, examples of sinuous, $3^{\text {rd }}$ antinode, oscillating wall jet, and impact point instabilities. For reference, these are runs $644,643,641$, and 631,with rescaled Reynolds numbers of 7,662; 5,788; 4,344 and 3,515, respectively. 
represent the onset of flow unsteadiness for the $60^{\circ}$ case. The clustering of symbols at an equivalent-jet diameter of about 3.5 is associated with unsteadiness onset that originates at the flow triple point for jets with a JPR greater than about 4. The second clustering of symbols between 6 and 7 equivalent-jet diameters is associated with unsteadiness onset that originates at the second antinode of flows with a $J P R$ of less than about 4 . The lone symbol with a rescaled Reynolds number of about 8,200 is actually from a run with a slightly overexpanded jet, with a $J P R$ of 0.8 , which exhibited a sinuous mode of transition. Single-shot PLIF images and the associated standard deviation images show examples of these three types of modes to transition on the right side of Fig. 10. In the case of the mid-JPR jet, the standard deviation image reveals instabilities originating from the second antinode. For the large $J P R$ jet, instabilities are seen to begin near the triple point and reflected shock/jet boundary intersection. Note that the impingement distance (the perpendicular distance from the nozzle exit to the center of the impingement plate) was $6.4 \mathrm{~cm}\left(2.5 \mathrm{in}\right.$.) for the $60^{\circ}$ case.

The model configuration with an oblique impingement angle of $45^{\circ}$ had an impingement distance of $9.5 \mathrm{~cm}$ (3.75 in.), which is $50 \%$ greater than that of the $60^{\circ}$ case. While the data in Fig. 11 show similar trends as in Fig. 10 , these trends are not as clear, owing partly to fewer cases exhibiting transitional behavior. The antinode instability is once again seen for two of the runs with $J P R$ less than 4, but in this case, the instability arises at the third antinode, rather than the second antinode. The increased distance to the impingement plate and the more oblique impingement angle may both contribute to this observed delay in the onset of unsteadiness. We postulate that the latter has the greater effect because a similar graph for the $90^{\circ}$ impingement case at the same impingement distance (see Fig. 12) reveals a significantly earlier average onset of unsteady behavior. This assertion is discussed in more detail below. An additional trend was observed in the behavior of the wall jet for several cases. Namely, oscillations were seen in the wall jet itself, and these oscillations were seen to contribute to the growth of instabilities in the flow. The impact point (where the jet boundary intersects the impingement plate) was also found to contribute to the amplification of flow instabilities. Single-shot PLIF images and the associated standard deviation images show examples of these four types of instability modes on the right side of Fig. 11.

2. Effect of Impingement Distance

The overall effect of impingement distance was found to be that smaller impingement distances resulted in the flow becoming unsteady sooner. However, as the data below will show, the downstream distance to the onset of unsteadiness was found to strongly depend on the location of certain key flow structures. In order to examine the effect of impingement distance quantitatively, data from all supersonic nozzle, $90^{\circ}$ impingement cases have been separated according to impingement distance and are shown in Fig. 12. As in the graphs above, grey lines connect data points from individual runs. By examining the length of these lines, a trend emerges as a function of impingement distance. Namely, the typical distance between the onset of flow unsteadiness and the onset of transition is seen to decrease as impingement distance decreases. For low JPR, the initial location of the onset of unsteadiness is seen to move from the third antinode to the second antinode. For high $J P R$, the nozzle lip is seen to emerge as a location where some unsteady behavior begins for the smallest impingement distance. In general, these flow features are seen to determine, to a large extent, where the flow initially becomes unsteady, whereas the impingement distance is seen to affect the distance which is then required for the flow to become transitional.

3. Comparison of Free vs. Impinging Jets

In an effort to summarize these findings, Fig. 13 contains graphs of the distance downstream at which normal impingement, supersonic nozzle flows become unsteady, transitional, or turbulent as a function solely of impingement distance. In addition to the data presented in Fig. 12, these graphs include data from free (nonimpinging) cases. The data have been graphed in terms of the reciprocal of impingement distance, such that free cases are defined as having an infinite impingement distance and appear at $1 / \mathrm{D}_{\mathrm{imp}}=0$. The data are separated into $J P R<3.5$ (on the left) and $J P R>3.5$ (on the right). Open symbols once again indicate locations along the impingement plate. On both graphs, two general trends emerge. First, the distance to unsteadiness (indicated by the yellow squares) for the free jet case is seen to occur somewhat further downstream than in any of the impinging jet cases. Second, the distance to transition (red triangles) or turbulence (brown squares) is in general seen to decrease for decreasing impingement distance. The presence or absence of an impingement target seems to determine where unsteadiness will first be manifest in a flow, with key flow features playing the dominant role for impinging jet cases, whereas impingement distance seems to control the location of the onset of transition and/or turbulence. 

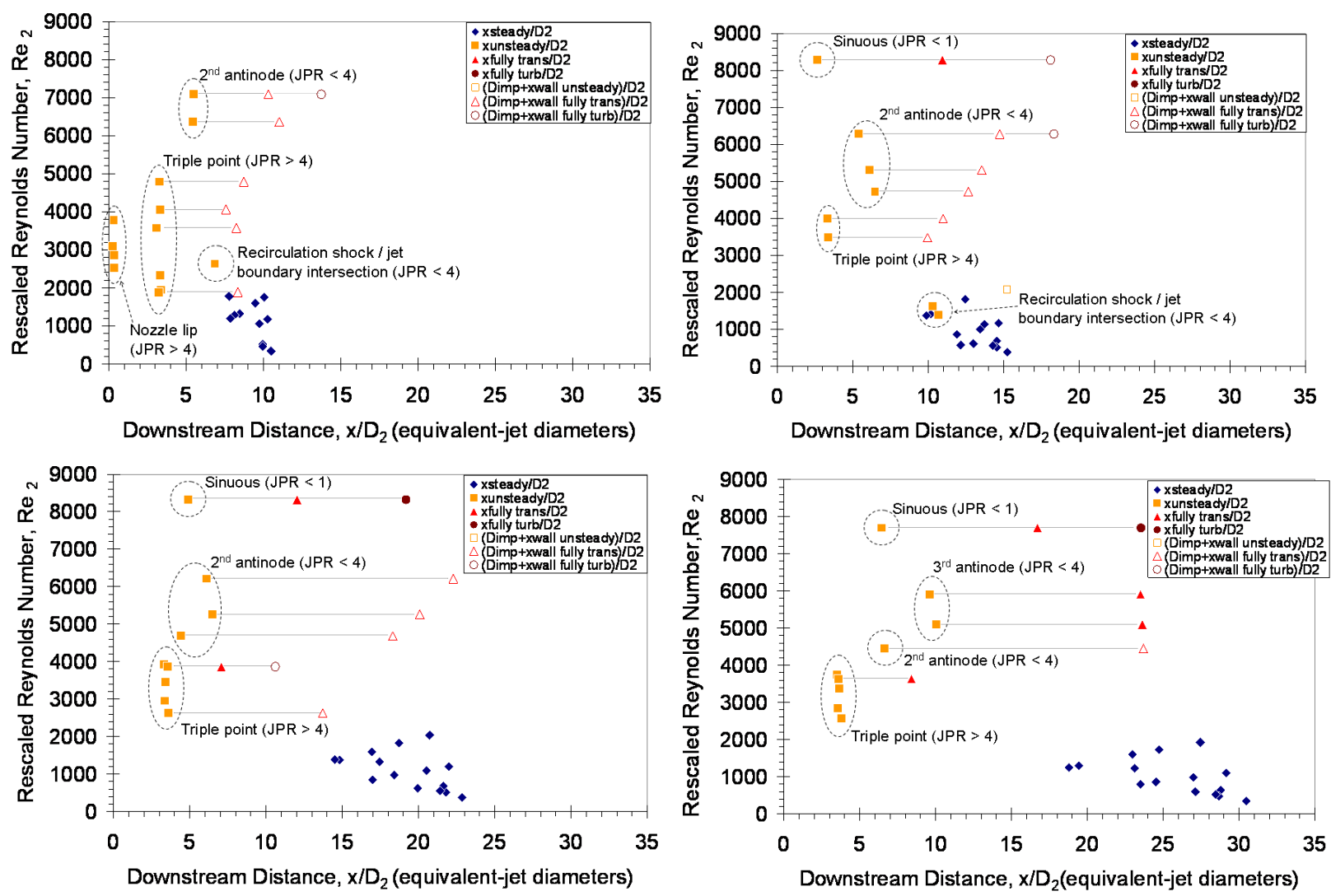

Figure 12: Effect of impingement distance at a normal $\left(\mathbf{9 0}^{\circ}\right)$ impingement angle. Data are for supersonic nozzle cases, and are separated according to impingement distance: $4.45 \mathrm{~cm}$ (1.75 in.), upper left; $6.35 \mathrm{~cm}(2.50$ in.), upper right; $9.53 \mathrm{~cm}$ (3.75 in.), lower left; $12.7 \mathrm{~cm}$ (5.00 in.), lower right. Distances are given in equivalent-jet diameters. Open symbols indicate locations along the face of the impingement plate. Light grey lines connect data points associated with the same run.
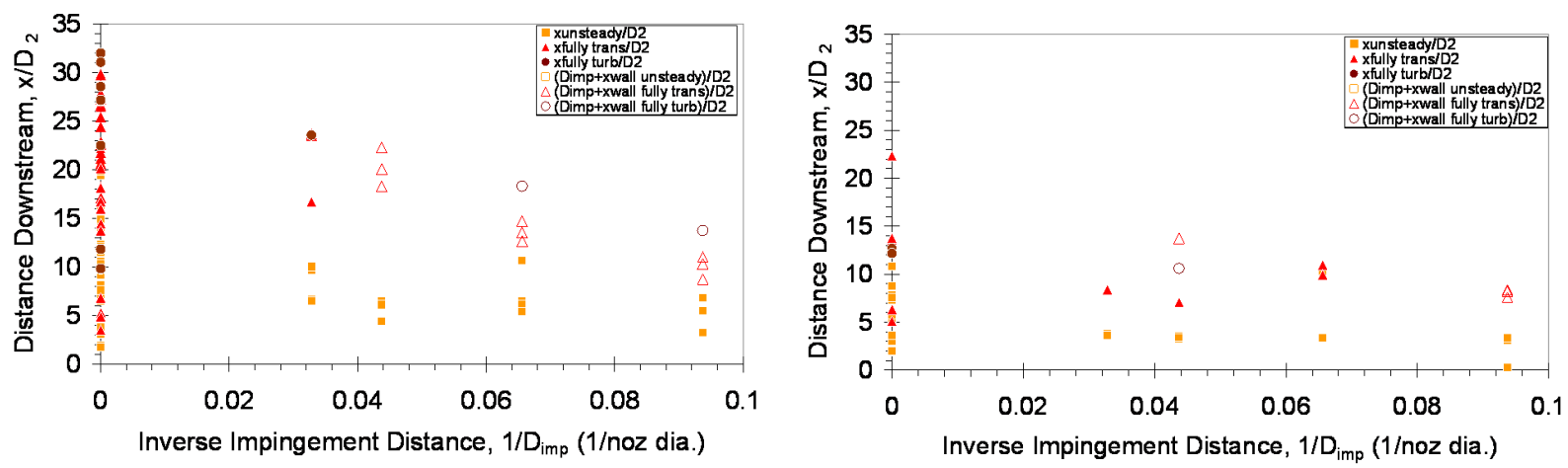

Figure 13: Effect of impingement distance on transition length (supersonic nozzle, $\theta_{\text {imp }}=90^{\circ}$ ). Graph on the left shows data from cases with $J P R<3.5$; graph on the right is for data with $J P R>3.5$. Free (non-impinging) cases are considered to have an infinite impingement distance $\left(D_{\text {imp }}=\infty ; 1 / D_{\text {imp }}=0\right)$.

Figure 13 contains data from all supersonic nozzle, $90^{\circ}$ impingement cases, as well as data from supersonic nozzle free jet cases which fell within the same range of rescaled Reynolds numbers and jet pressure ratio as the impinging data set. However, due to variability in target (i.e. setpoint) versus actual (i.e. measured) plenum conditions, not all impinging cases shown have a suitably comparable free jet case, and vice versa. The impinging data were further reduced to remove from consideration any data point which did not have a comparable free jet case 
(where a "comparable case" was arbitrarily defined to be a case for which both $\operatorname{Re}_{2}$ and $J P R$ were within $25 \%$ of the values in the impinging case).

Direct comparisons were then made and the reduction factor in the distance to unsteadiness, transition, or turbulence was calculated. The reduction factor was defined to be the factor by which the distance to unsteadiness, transition or turbulence was reduced in a given impingement case compared to the corresponding free jet case. For instance, if a jet was found to become fully turbulent in the free jet case after 28 equivalent-jet diameters (i.e. $\mathrm{x}_{\text {fully }}$ turb $/ \mathrm{D}_{2}=28$ ), and if this same measurement indicated 16 equivalent-jet diameters in the impinging jet case, then the reduction factor would be $28 \div 16=1.75$.

Figure 14 contains a summary of the average reduction factor at the four impingement distances used for $90^{\circ}$ impingement cases, with high $(>3.5$, black triangles) and low $(<3.5$, grey diamonds) jet pressure ratios graphed separately. Note that for high $J P R$ cases, the target was at the same physical distances from the nozzle exit, but was effectively closer if $D_{\text {imp }}$ is given in terms of the number of jet diameters (the horizontal axis on this graph is given in units of equivalent-jet diameters). Horizontal error bars indicate one standard deviation in the average value of $\mathrm{D}_{\mathrm{imp}} /$ $\mathrm{D}_{2}$ for the cases included in each data point (where no error bars are seen, the error bars are smaller than the size of the data points themselves). Vertical error bars indicate plus and minus one standard deviation in the average measured distances. Two of the low JPR cases had only a single comparison point (one free jet

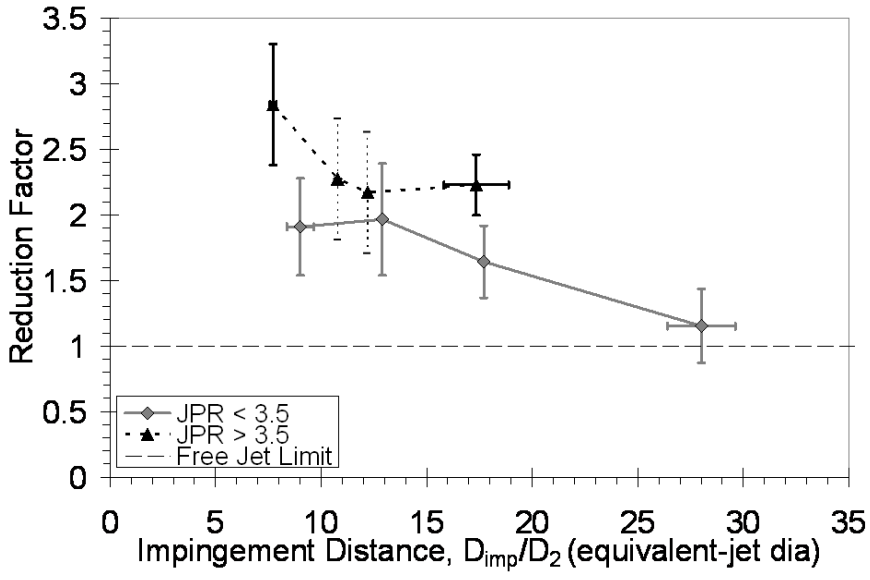

Figure 14. Reduction factor in the distance to unsteady jet behavior as a function of impingement distance. Data are for supersonic nozzle, $90^{\circ}$ impingement cases. case and one impinging jet case), so no standard deviation could be computed. For these two data points, dashed error bars indicate the maximum standard deviation of the other measurements as an estimate of the expected error in these measurements. A dashed horizontal line indicates a reduction factor of 1 , which is the limit the data are expected to approach as the impingement configuration becomes a free jet configuration.

Figure 14 shows that an impingement target at 28 equivalent-jet diameters $(30.5$ nozzle diameters, $12.7 \mathrm{~cm}, 5.0$ inches) has only a small effect on the unsteady behavior of a jet for low JPR. As the impingement distance decreases, however, the effect of the target increases. At the two closest impingement distances of 9.0 and 12.9 equivalent-jet diameters (10.7 noz. dia., $4.4 \mathrm{~cm}, 1.75$ in. and 15.2 noz. dia., $6.4 \mathrm{~cm}, 2.5$ in., respectively), the measured distances to unsteadiness, transition, and/or turbulence were found to decrease by a factor of about 2 , relative to the free jet case, for low $J P R$ cases. For high $J P R$ cases, the presence of the impingement target appears to have had a slightly greater effect, with the reduction factor ranging from 2.8 to 2.2 . Recall that information (in the form of pressure waves) about the presence of the impingement target can only propagate upstream through subsonic regions of the jet flow. For high $J P R$ jets, this includes the ambient gas around the jet, subsonic parts of the jet boundary, and subsonic regions of the jet core. For low JPR jets, only these first two regions are usually applicable. Perhaps the difference in these feedback mechanisms for jets with different flow structures is the underlying reason for the distinction between reduction factors for high and low JPR jets.

Many of the aforementioned trends in the data are apparent by a visual comparison between graphs of all impinging jet cases versus all free jet cases. Figure 15 shows data points for all sonic nozzle runs, with all free jet cases on the left and all impinging jet cases on the right. Notice that for free jet cases, the data show an inverse relationship between rescaled Reynolds number and distance to unsteadiness, transition, and turbulence (lines indicated power law fits to the data). In other words, the higher the Reynolds number, the shorter the distance to unsteadiness, transition, and/or turbulence. Now notice that this trend is not readily apparent for impinging jet cases. Instead, data points are seen to cluster around downstream locations corresponding to certain flow features. Also notice that for impinging jet cases, runs with rescaled Reynolds numbers below about 1,800 are observed to remain laminar across the entire field of view, whereas the free jet results do not indicate a Reynolds number threshold for unsteady behavior, but rather indicate a functional dependence between the rescaled Reynolds number and the downstream distance. The clustering in the impinging cases of unsteady points near an equivalent-jet diameter of about 1 was shown above to be largely associated with the triple point instability mode for flows with $J P R$ greater 
than about 3. Note also that no cases were seen to become fully transitional prior to about 1.5 equivalent-jet diameters downstream of the nozzle exit. For the pressure ratios and impingement distances studied in these experiments, the majority of the sonic nozzle impinging jet cases that did become unsteady, transitional, or turbulent were observed to do so prior to the impingement target (as evidenced by the paucity of open symbols in the impinging jet data. Finally, notice that the overall effect of the presence of an impingement target is to decrease the distance to unsteadiness, transition, and/or turbulence.

Figure 16 contains the corresponding graphs for supersonic nozzle cases. Again, an inverse relationship between rescaled Reynolds number and downstream distance is seen for free jet cases. For impinging jet cases, flows with a rescaled Reynolds number of less than about 1,800 were observed to remain steady and laminar, as in the sonic nozzle results, with the exception of two runs. The two seemingly anomalous runs were both found to have recirculation bubbles that exhibited unsteadiness and appeared to be close to a staging behavior transition. (See Ref. 16 for a detailed discussion of recirculation bubble formation and associated staging behavior.) A similar division (i.e. a rescaled Reynolds number below which all points were observed to remain steady and laminar) is seen in the supersonic free jet cases, but at a slightly higher rescaled Reynolds number of about 2,600 (a result reported previously in Ref. 10). This comparison between the free and impinging cases implies that the presence of the impingement target does in fact encourage the growth of instabilities in the flow, lowering the threshold below which flows remain laminar and steady. As discussed in more detail above for individual impingement configurations, two clusterings are evident in the unsteady impinging jet symbols of Fig. 16. The first is for equivalent-jet diameters of less than 1; the second is between 3 and 4 diameters. These correspond to nozzle lip and triple point instabilities, respectively.

Upon further consideration, the problem may need to be redefined for making measurements of the location of flow unsteadiness for once the flow has entered the impingement region. Flow structures play a key role in the
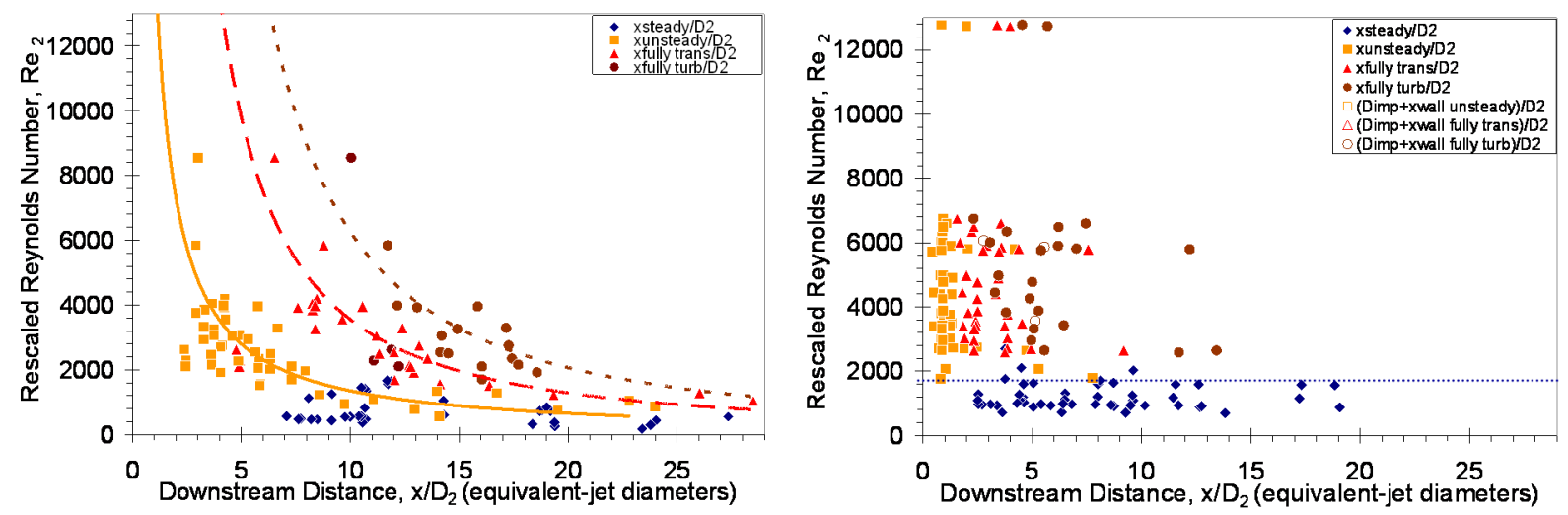

Figure 15. Sonic nozzle: comparison of free (left) and impinging (right) jets.
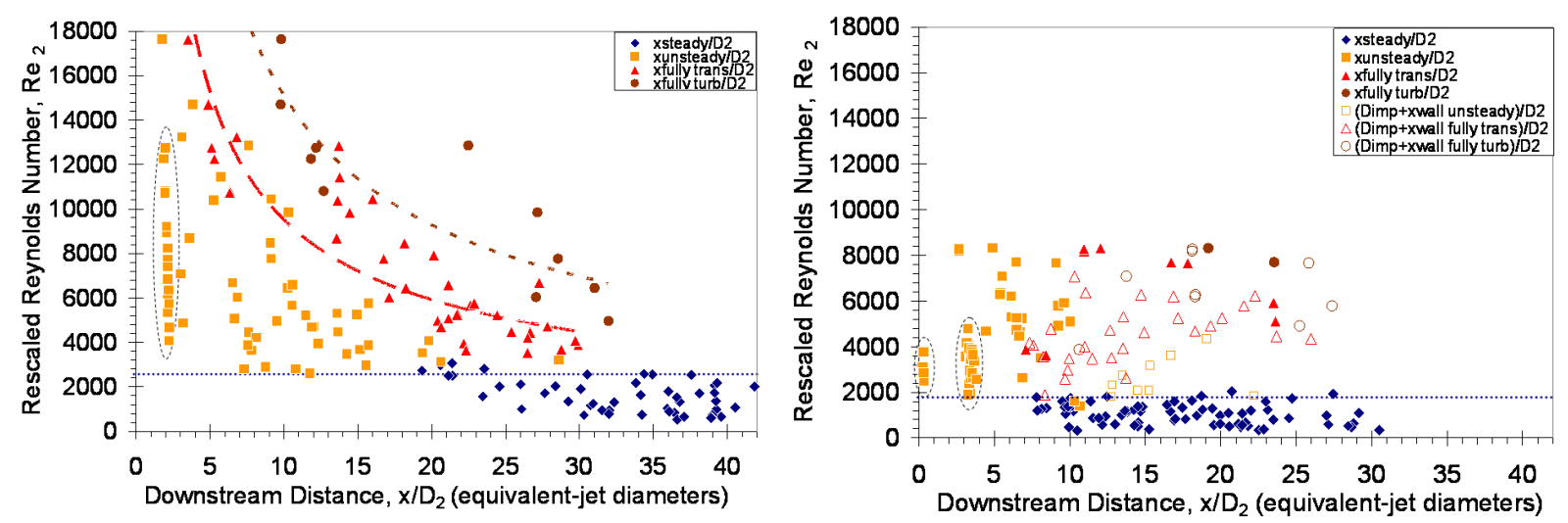

Figure 16. Supersonic nozzle: comparison of free (left) and impinging (right) jets. 
development of flow instabilities upstream of the impingement target, and so a length scale based on the jet diameter is appropriate in the free jet region. After impingement, however, additional constraints are imposed upon the flow and so the factors most relevant to the transitional behavior of the flow may change significantly as the flow becomes a wall jet. Since the flow then becomes essentially a diverging flow over a flat plate, the physical distance along the plate may, for example, be a more suitable length scale for quantifying transition location in this region.

The overall effect of the presence of the impingement target is again seen to be a decrease in the distance to unsteadiness, along with a clustering of data points around distances corresponding to major flow features. Interestingly, the majority of flows in these tests were observed to become unsteady at the triple point. One potential explanation for this behavior may be a result of the subsonic velocities which presumably exist in the jet core, downstream of the Mach disk, in cases with high jet pressure ratios. Disturbances to the pressure field caused by the presence of the impingement target could propagate upstream in a subsonic jet core, or through the subsonic ambient gas surrounding the jet. Since such disturbances would be prevented from traveling upstream in supersonic regions of the flow, the locations which were observed to be sources of amplification of flow instabilities-namely, the nozzle lip, the triple point, and the intersection of the reflected shock with the jet boundary-are logical locations where one might expect these disturbances to become concentrated. Measurements of the velocity field, particularly the axial component of velocity, would be helpful in either refuting or strengthening this explanation of the observed flow behavior.

\section{Conclusion and Summary Remarks}

PLIF images have been used to visualize impinging underexpanded jet flows. They have provided detailed information about free and impinging jet flow structures not attainable by other methods and have allowed determination of the laminar, unsteady, or turbulent state of the flow. The insights into flow structure characteristics provided by PLIF images have helped to elucidate the flow structures associated with the growth of instabilities in underexpanded jets and to quantify criteria for the onset of unsteadiness and the transition to turbulence. Trends have been identified that indicate that the behavior of impinging jet flows displays fundamentally different characteristics than that of comparable free jet flows. The distance downstream of the nozzle exit at which a free, underexpanded jet flow exhibits unsteady, transitional, or turbulent behavior had previously been shown to be strongly dependent on the rescaled Reynolds number of the flow, for both sonic and supersonic nozzle flows. ${ }^{9,10,14}$ The present study has shown that the presence of an impingement target not only reduces the distance required for a flow to become unsteady, transitional, or turbulent, but also increase the significance of key flow features in determining the location of the onset of these behaviors. Normal $\left(90^{\circ}\right)$ impingement was shown to be more perturbative than oblique $\left(45^{\circ}\right.$ or $\left.60^{\circ}\right)$ impingement as evidenced by greater amplification and growth of flow instabilities in $90^{\circ}$ cases. It has been proposed that subsonic regions of the flow provide a feedback mechanism via which the presence of the impingement target can influence and promote the development of instabilities upstream of the impingement region. Furthermore, such instabilities appear to become concentrated around flow features associated with locations bordering both subsonic and supersonic regions of the flow. For the impingement distances and angles of this study, the data indicate that location of the onset of flow unsteadiness can be reduced in the presence of an impingement target by a factor of up to 2.8 compared to free jet cases. Axial velocity measurements are recommended as a means of garnering further insight into the mechanisms of transition in underexpanded jet flows.

\section{Acknowledgments}

The authors wish to acknowledge the collaborative input of Scott Halloran, formerly with The Boeing Company, Don Picetti of The Boeing Company, and Chris Glass of NASA Langley Research Center, as well as the technical assistance of Stephen Jones, and Paul Tucker, also of NASA Langley Research Center. They also wish to acknowledge the image processing work done by Aiyana Garcia, a graduate physics student from The College of William and Mary and a NASA GSRP (Graduate Student Researchers Program) student. This work was funded as part of the Shuttle Return to Flight effort through Chuck Campbell of Johnson Space Center and Tom Horvath of NASA Langley Research Center, as well as through the ongoing support of the Hypersonics Program at NASA Langley Research Center. 


\section{References}

${ }^{1}$ Anderson, John D., Jr., Modern Compressible Flow: With Historical Perspective, $3^{\text {rd }}$ ed., McGraw-Hill, Boston, 2003.

${ }^{2}$ Love, E. S., C. E. Grigsby, and L. P. Lee, "Shape of Initial Portion of Boundary of Supersonic Axisymmetric Free Jets at Large Pressure Ratios," NACA TN 4195, January 1958.

${ }^{3}$ Love, E. S., C. E. Grigsby, L. P. Lee, and M. J. Woodling, "Experimental and theoretical studies of axisymmetric free jets," NASA Technical Report R-6, 1959.

${ }^{4}$ Donaldson, C. D., and R. S. Snedeker, “A study of free jet impingement. Part 1. Mean properties of free and impinging jets," J. Fluid Mech. 45: Part 2, 1971, pp. 281-319.

${ }^{5}$ Donaldson, C. D., R. S. Snedeker, and D. P. Margolis, “A study of free jet impingement. Part 2. Free jet turbulent structure and impingement heat transfer," J. Fluid Mech. 45: Part 3, 1971, pp. 477-512.

${ }^{6}$ Henderson, Brenda, "The connection between sound production and jet structure of the supersonic impinging jet," Journal of the Acoustical Society of America 111 (2), February 2002, pp. 735-747.

${ }^{7}$ Lamont, P.J., and B.L. Hunt, "The Impingement of Underexpanded Axisymmetric Jets on Perpendicular and Inclined Flat Plates," Journal of Fluid Mechanics 100, 1980, pp. 471-511.

${ }^{8}$ Belan, Marco, Sergio De Ponte, and Daniela Tordella, "Determination of Density and Concentration from Fluorescent Images of a Gas Flow," Experiments in Fluids 45 (3), September 2008, pp. 501-511.

${ }^{9}$ Wilkes, Jennifer A., Paul M. Danehy, and Robert J. Nowak "Fluorescence Imaging Study of Transition in Underexpanded Jets," Proceedings of the 21st International Congress on Instrumentation in Aerospace Simulation Facilities (ICIASF) [CDROM], Sendai, Japan, 29 August - 1 September 2005, pp. 1-8.

${ }^{10}$ Inman, Jennifer A. (Wilkes), Paul M. Danehy, Robert J. Nowak, and David W. Alderfer, "Identification of Instability Modes of Transition in Underexpanded Jets," AIAA-2008-4389, 38th AIAA Fluid Dynamics Conference and Exhibit, Seattle, WA, 23-26 June 2008.

${ }^{11}$ Yüceil, K. Bülent, and M. Volkan Ötügen, "Scaling parameters for underexpanded supersonic jets," Physics of Fluids 14 (12), December 2002, pp. 4206-4215.

${ }^{12}$ White, F. M., Viscous Fluid Flow, $2^{\text {nd }}$ ed., McGraw-Hill, Inc., New York, 1991.

13 Palma, P.C., Laser-Induced Fluorescence Imaging in Free-Piston Shock Tunnels. Ph.D. thesis, Australian National University, submitted May 1998, revised February 1999.

${ }^{14}$ Inman, Jennifer A., "Fluorescence Imaging Study of Free and Impinging Supersonic Jets: Jet Structure and Turbulent Transition," Ph.D. Dissertation, Department of Physics, The College of William and Mary, Williamsburg, VA, 2007.

15 Wilkes, Jennifer A., Christopher E. Glass, Paul M. Danehy, and Robert J. Nowak, "Fluorescence Imaging of Underexpanded Jets and Comparison with CFD," AIAA-2006-0910, $44^{\text {th }}$ AIAA Aerospace Sciences Meeting and Exhibit, Reno, NV, 9-12 January 2006.

${ }^{16}$ Inman, Jennifer A. (Wilkes), Paul M. Danehy, Robert J. Nowak, and David W. Alderfer, "Fluorescence Imaging Study of Impinging Underexpanded Jets," AIAA 2008-619, 46th AIAA Aerospace Sciences Meeting and Exhibit, Reno, NV, 7-10 January 2008.

${ }^{17}$ Danehy, P.M., A.P. Garcia, S. Borg, A.A. Dyakonov, S.A. Berry, J.A. (Wilkes) Inman, and D.W. Alderfer (8-11 Jan 2007). "Fluorescence visualization of hypersonic flow past triangular and rectangular boundary-layer trips." $45^{\text {th }}$ AIAA Aerospace Sciences Meeting and Exhibit, Reno, NV. AIAA 2007-0536. 